\title{
Symmetry restoration at high-temperature in two-color and two-flavor lattice gauge theories
}

\author{
Jong-Wan Lee, ${ }^{a, b, c}$ Biagio Lucini ${ }^{a}$ and Maurizio Piai ${ }^{a}$ \\ ${ }^{a}$ Department of Physics, College of Science, Swansea University, \\ Singleton Park, SA2 8PP, Swansea, Wales, U.K. \\ ${ }^{b}$ Department of Physics, Pusan National University, \\ Busan 46241, Korea \\ ${ }^{c}$ Extreme Physics Institute, Pusan National University, \\ Busan 46241, Korea \\ E-mail: jwlee823@pusan.ac.kr, b.lucini@swansea.ac.uk, \\ m.piai@swansea.ac.uk
}

AbStract: We consider the $\mathrm{SU}(2)$ gauge theory with $N_{f}=2$ flavors of Dirac fundamental fermions. We study the high-temperature behavior of the spectra of mesons, discretizing the theory on anisotropic lattices, and measuring the two-point correlation functions in the temporal direction as well as screening masses in various channels. We identify the (pseudo-)critical temperature as the temperature at which the susceptibility associated with the Polyakov loop has a maximum. At high temperature both the spin- 1 and spin-0 sectors of the light meson spectra exhibit enhanced symmetry properties, indicating the restoration of both the global $\mathrm{SU}(4)$ and the axial $\mathrm{U}(1)_{A}$ symmetries of the model.

KeYwords: Global Symmetries, Lattice Quantum Field Theory, Quark-Gluon Plasma, Technicolor and Composite Models

ArXiv ePrint: 1701.03228 


\section{Contents}

1 Introduction 1

2 The model: symmetry considerations 3

2.1 Composite states: scalars 4

$\begin{array}{ll}2.2 \text { Composite states: vectors } & 6\end{array}$

3 Numerical results: anisotropic lattice $\quad 7$

$\begin{array}{lll}3.1 & \text { Lattice action } & 7\end{array}$

$\begin{array}{llr}3.2 & \text { Simulation details } & 9\end{array}$

$\begin{array}{lll}3.3 & \text { Gauge anisotropy } & 10\end{array}$

$\begin{array}{lll}3.4 & \text { Fermion anisotropy } & 12\end{array}$

$\begin{array}{ll}3.5 & \text { Tuning results } \\ \end{array}$

4 Numerical results: finite temperature $\quad \mathbf{1 5}$

$\begin{array}{lll}4.1 & \text { Deconfinement crossover } & 17\end{array}$

$\begin{array}{ll}4.2 & \text { Temporal correlation functions } \\ & 17\end{array}$

$\begin{array}{ll}4.3 & \text { Spatial correlation functions } 20\end{array}$

5 Discussion $\quad 25$

$\begin{array}{ll}\text { A Spinors and global symmetries } & 26\end{array}$

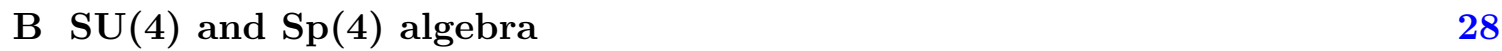

C Fit results of renormalized parameters 31

\section{Introduction}

We consider the SU(2) gauge theory with $N_{f}=2$ flavors of Dirac fundamental fermions, and study the finite-temperature behavior by using numerical methods based on formulating the theory on anisotropic lattices. The main purpose of this work is to collect evidence that the global symmetries of the model are implemented $\grave{a}$ la Wigner at high-temperature, where the condensate breaking global symmetry is expected to melt and the global symmetries to be linearly realized.

This model has been considered before in three different contexts, as it represents the prototype of non-trivial gauge theory in which lattice numerical methods have concrete potential to provide useful information about the dynamics of the underlying theory. First of all, it is a useful toy model for the study of generalizations of Quantum Chromo-Dynamics (QCD) at finite temperature $T$ and finite chemical potential $\mu$. One trivial reason for this is that the number of fundamental degrees of freedom is smaller than for two-flavor 
QCD, making the numerical treatment easier. Most importantly though, the fundamental representation of $\mathrm{SU}(2)$ is pseudo-real, and hence there is no sign problem. It is then possible to study the phase diagram of the model in the $(T, \mu)$-plane, and to apply numerical techniques to extract its detailed structure. For an incomplete list of useful references on the subject see $[1-3]$.

A second context in which this model is important is that of traditional technicolor (TC) [4-12]. The choice of SU(2) with 2 fundamental Dirac fermions yields the minimal model such that one can embed the electro-weak $\mathrm{SU}(2)_{L} \times \mathrm{U}(1)_{Y}$ group of the Standard Model of particle physics (SM) within the global symmetries of the matter field content. One expects spontaneous symmetry breaking to arise dynamically at the scale $\Lambda$, hence providing a natural way to implement the Higgs mechanism for giving mass to the electroweak bosons within a fundamental theory. Aside from the fact that, once more, the small number of degrees of freedom makes practical applications amenable to numerical treatment, the fact that the field content is minimal also minimizes the potentially problematic contributions to precision parameters such as the oblique $S$ and $T$ as defined by Peskin and Takeuchi [13], that on the basis of perturbative arguments one expects to grow with $N_{f}$ and $N_{c}$, and that are not dynamically suppressed when one identifies $\Lambda$ with the electroweak scale $v_{W} \sim 246 \mathrm{GeV}$. The dynamics preserves a custodial SU(2) that further suppresses the $T$ parameter, as the underlying masses of the fermions vanish.

The model has received some attention in a third context [14-27], as a concrete realization of the idea of Higgs compositeness [28]. This is a quite distinct framework in respect to traditional TC. The underlying dynamics is the same, being based upon a gauge theory with a given global symmetry, for which one expects the formation of a non-trivial symmetry-breaking condensate. Yet, one chooses to embed the electroweak gauge group into the global symmetry group of the theory in such a way that the fermion condensate does not break it. ${ }^{1}$ The long-distance behavior of the theory is hence captured by an Effective Field Theory (EFT) that includes the SM gauge theory, supplemented by a set of light, composite pseudo-Goldstone bosons arising at the scale $\Lambda$, a subset of which is interpreted as the Higgs doublet field.

The gauging of the SM group explicitly breaks the global symmetries, and hence provides a potential for the Higgs fields. Additional ingredients, not arising from the SU(2) fundamental gauge theory, are invoked in order to drive spontaneous symmetry breaking in the Higgs sector, which ultimately yields electro-weak symmetry breaking (EWSB) at the scale $v_{W} \ll \Lambda$. For example, one has to introduce a mechanism to give mass to the SM fermions, which requires coupling the Higgs field to the quarks and leptons. It is well known that, as a byproduct of doing so, the theory yields radiative corrections to the Higgs potential due to loops of the top quark, naive estimates of which show that they can destabilize the minimum of the Higgs potential. In the following we will not discuss any of these points, related to realistic model-building in the electro-weak sector.

The reason why composite scenarios are viable within this model originates from the pseudo-real nature of the fundamental representation of $\mathrm{SU}(2)$. In particular, in

\footnotetext{
${ }^{1}$ We ignore the problem of vacuum alignment [29].
} 
the presence of two Dirac fermions, the global symmetry of the Lagrangian is enhanced from the $\mathrm{U}(1)_{A} \times \mathrm{U}(1)_{B}^{t} \times \mathrm{SU}(2)_{L}^{t} \times \mathrm{SU}(2)_{R}^{t}$ global symmetry of QCD and TC to a $\mathrm{U}(1)_{A} \times \mathrm{SU}(4)$ global symmetry, and the condensate breaks it to the $\mathrm{Sp}(4)$ subgroup. Excluding for the time being the anomalous $\mathrm{U}(1)_{A}$ from the discussion, this yields 5 (pseudo-)Goldstone bosons, that form a multiplet of the unbroken $\mathrm{Sp}(4) \sim \mathrm{SO}(5)$. The gauging of $\mathrm{SU}(2)_{L} \times \mathrm{U}(1)_{Y} \subset \mathrm{SO}(4) \subset \mathrm{Sp}(4)$ splits the 5 into a 4 of $\mathrm{SO}(4)$, which is identified with the Higgs doublet, and an additional singlet, that may have important phenomenological implications.

In this paper, we compute the masses of the composite (meson) states created and annihilated by operators of the form $\bar{Q} \Gamma Q$, with $\Gamma=1, \gamma_{5} \cdots$, and discuss their dependence on temperature $T .^{2}$ In particular we track how the mass-splittings between parity partners change by going to high-temperature. In order to do so, we formulate the theory on anisotropic lattices, and use Monte Carlo methods to extract the spectral masses as a function of $T$. We are looking for clear signals of the restoration in the thermal bath of the much larger global symmetry of the underlying theory. This is the first step of a more ambitious and long-term program, which we envision will include also the study of the effects due to the presence of explicit symmetry-breaking terms, in particular due to the chemical potential $\mu$, and to the weakly-coupled gauging of the SM electroweak group.

The paper is organized as follows. In section 2 we describe the model and summarize effective field theory and symmetry arguments that play a role in the rest of the paper. In section 3 we describe the lattice set-up used in the numerical calculations, particularly by explaining in details how the bare parameters are tuned in the presence of anisotropic lattices. In section 4 we report our results, which we critically discuss in section 5. Appendix A contains some useful notation about spinors, and we show explicitly how the enhanced global symmetry emerges. In appendix B we summarize the algebraic properties of $\mathrm{SU}(4)$ and $\mathrm{Sp}(4)$, by providing an explicit example of generators for $\mathrm{SU}(4)$. Examples of the renormalized versus bare parameters are given in appendix $\mathrm{C}$.

\section{The model: symmetry considerations}

The matter field content consists of two (massive) Dirac fermions $Q^{i a}$, where $a=1,2$ is the $\mathrm{SU}(2)$ color index and $i=1,2$ the flavor index. The covariant derivative is

$$
\left(D_{\mu} Q^{i}\right)^{a}=\partial_{\mu} Q^{i a}+i g V_{\mu}^{A}\left(T^{A}\right)_{b}^{a} Q^{i b},
$$

with $V_{\mu}^{A}$ the gauge fields, $g$ the coupling, and $T^{A}$ the generators of $\mathrm{SU}(2)$ obeying $\operatorname{Tr} T^{A} T^{B}=\frac{1}{2} \delta^{A B}$, so that $T^{A}=\tau^{A} / 2$. The Lagrangian density is

$$
\mathcal{L}=i \overline{Q^{i}}{ }_{a} \gamma^{\mu}\left(D_{\mu} Q^{i}\right)^{a}-m \overline{Q^{i}}{ }_{a} Q^{i a}-\frac{1}{2} \operatorname{Tr} V_{\mu \nu} V^{\mu \nu},
$$

where the summations over flavor index $i=1,2$ and color index $a=1,2$ are understood, and where the field-strength tensors are defined in terms of the gauge bosons as $V_{\mu \nu} \equiv$ $\partial_{\mu} V_{\nu}-\partial_{\nu} V_{\mu}+i g\left[V_{\mu}, V_{\nu}\right]$.

\footnotetext{
${ }^{2}$ We refer the reader to the works in [30-32]: while these papers study $\mathrm{SU}(3)$ gauge theory, some of their results and ideas play a role in the present paper.
} 


\begin{tabular}{|c|c|c|}
\hline Fields & $\mathrm{SU}(2)$ & $\mathrm{SU}(4)$ \\
\hline$V_{\mu}$ & 3 & 1 \\
$q$ & 2 & 4 \\
\hline$\Sigma_{0}$ & 1 & 6 \\
$M$ & 1 & 6 \\
\hline
\end{tabular}

Table 1. The field content of the model. $V_{\mu}$ are gauge bosons, $q$ are two-component spinors, $\Sigma_{0}$ is a composite scalar, $M$ a scalar spurion.

We collect in appendix A and B several useful relations between 2-component spinors $q$ and 4-component spinors $Q$, as well as details about the algebra of $\mathrm{SU}(4)$ and $\mathrm{Sp}(4)$ (see also [14-27]). The global symmetry acting on the matter fields is $\mathrm{U}(1)_{A} \times \mathrm{SU}(4)$, and we explicitly list the transformation properties of the fields in table 1 . It is convenient to define:

$$
\Sigma_{0}^{n m}=\sum_{a b} \epsilon_{a b} q^{n a T} \tilde{C} q^{m b}
$$

and to write the mass explicitly as a matrix $M \equiv m \Omega$, with $\Omega$ the symplectic matrix in eq. (B.1). The index $n, m=1, \cdots, 4$ and $\tilde{C}=-i \tau^{2}$ acts on spinor indexes. In the lower half of table 1 we list the transformation properties of the composite field $\Sigma_{0}$, as well as the (symmetry-breaking) spurion $M$.

In the body of the paper, we will describe the finite-temperature properties of composite states that we identify with the pions $\pi, \rho$ vector, $a_{1}$ axial-vector, and $a_{0}$ scalar mesons. In the rest of this section, we summarize the basic properties of these objects, using the language of effective field theory (EFT). What results is a Lagrangian density that includes potentially heavy and strongly-coupled degrees of freedom, and hence does not yield a calculable weakly-coupled low-energy EFT in the usual sense. We use this language to guide our book-keeping exercise, focused on classifying the physical particles, their quantum numbers, and the degeneracies - in particular the difference of mass between the $\rho$ and $a_{1}$ vectors and between the $\pi$ and $a_{0}$ scalars - that are consequences only of the symmetry structure of the theory and its vacuum.

\subsection{Composite states: scalars}

In the low-energy EFT description, the real antisymmetric field $\Sigma$ transforms as

$$
\Sigma \rightarrow U \Sigma U^{T}
$$

under the action of an element $U$ of $\mathrm{SU}(4)$. The $\operatorname{VEV}\langle\Sigma\rangle \propto \Omega$ breaks $\mathrm{SU}(4)$ to the $\mathrm{Sp}(4)$ subgroup. The generators $T^{A}$ with $A=1, \cdots, 5$ are broken, while $T^{A}$ with $A=6, \cdots, 15$ are unbroken. For instance, see eq. (B.4) in appendix B.

In terms of the matrix-valued $\pi(x)=\sum_{A=1}^{5} \pi^{A}(x) T^{A}$, the convenient parameterization

$$
\Sigma=e^{\frac{i \pi}{f}} \Omega e^{\frac{i \pi^{T}}{f}}=e^{\frac{2 i \pi}{f}} \Omega=\Omega e^{\frac{2 i \pi^{T}}{f}},
$$


automatically satisfies the non-linear constraint $\Sigma^{\dagger} \Sigma=\mathbb{I}_{4}$. The leading-order term of the low-energy EFT is

$$
\begin{aligned}
\mathcal{L}_{0} & =\frac{f^{2}}{4} \operatorname{Tr}\left\{\partial_{\mu} \Sigma\left(\partial^{\mu} \Sigma\right)^{\dagger}\right\} \\
& =\operatorname{Tr}\left\{\partial_{\mu} \pi \partial^{\mu} \pi\right\}+\frac{1}{3 f^{2}} \operatorname{Tr}\left\{\left[\partial_{\mu} \pi, \pi\right]\left[\partial^{\mu} \pi, \pi\right]\right\}+\cdots
\end{aligned}
$$

The pion fields are canonically normalized and hence $f=f_{\pi}$ is the pion decay constant.

The quark mass is incorporated in the EFT by adding the symmetry-breaking term

$$
\mathcal{L}_{m}=-\frac{v^{3}}{4} \operatorname{Tr}\{M \Sigma\}+\text { h.c. }=2 m v^{3}-\frac{m v^{3}}{f^{2}} \operatorname{Tr} \pi^{2}+\cdots .
$$

The expansion in pion fields confirms that the 5 pions are still degenerate, if not massless, in the presence of the explicit breaking given by the Dirac mass for the fermions, with

$$
m_{\pi}^{2} f_{\pi}^{2}=m v^{3}
$$

The degeneracy of the five pions is a consequence of the unbroken $\mathrm{Sp}(4) \sim \mathrm{SO}(5)$ symmetry. The spurion $M$ formally transforms as $M \rightarrow U^{*} M U^{\dagger}$, so that if it were promoted to a field then $\mathcal{L}_{m}$ would be manifestly invariant under the full SU(4) symmetry.

Here we pause to make two general observations. In the context of composite-Higgs models, the presence of a (small) mass term for the quarks is allowed, contrary to the TC case. While in the latter the quark mass explicitly breaks the gauge symmetries, in the composite-Higgs case the SM gauge group is a subgroup of $\mathrm{Sp}(4)$, and hence the term in eq. (2.8) does not break it. The distinction between TC and composite-Higgs cases reduces (in the massless case) to a vacuum alignment issue driven by the weak gauging of the $\mathrm{SU}(2)_{L} \times \mathrm{U}(1)_{Y}$ symmetry. In the presence of a mass of the form in eq. (2.8), this problem has a trivial solution: the mass $m$ stabilizes the composite-Higgs vacuum. Yet, some caution is in order: if $m_{\pi}$ is large, it might become impossible to induce electro-weak symmetry breaking. We leave these and similar issue out of this study (see [33]), as in our numerical work all calculations are done with the $\mathrm{SU}(2)$ theory in isolation.

To describe the regime in which the symmetry is restored, which is expected to be realized at high temperature, we remove the non-linear constraint, and hence replace $\Sigma$ by the field $H \sim 6$, that transforms as a complete antisymmetric representation of $\mathrm{SU}(4)$. The kinetic term is

$$
\mathcal{L}_{H}=\frac{1}{2} \operatorname{Tr} \partial_{\mu} H \partial^{\mu} H
$$

The Lagrangian density contains a potential as any arbitrary function $V\left(H^{\dagger} H\right)$ is allowed by the symmetries. The minimization of $V$ yields the identification $\langle H\rangle=\frac{f}{\sqrt{2}}\langle\Sigma\rangle=\frac{f}{\sqrt{2}} \Omega$. The small fluctuations of $H$ are parameterized in terms of the 5 pion fields along the broken directions, plus an additional real scalar $\sigma$ :

$$
H=\frac{f+\sigma}{\sqrt{2}} \Sigma=\frac{f+\sigma}{\sqrt{2}} e^{\frac{2 i \pi}{f}} \Omega,
$$


where the normalizations are chosen so that all the fields have canonical kinetic terms. Unconstrained by symmetry considerations, the scalar $\sigma$ (singlet of $\operatorname{Sp}(4)$ ) is expected to have a large mass $m_{\sigma}$, and in general decay fast to pions.

Besides the $\mathrm{SU}(4) \rightarrow \mathrm{Sp}(4)$ breaking, the vacuum also induces the breaking of the (anomalous) $\mathrm{U}(1)_{A}$. To discuss it, we need to promote $H$ a complex field, hence doubling the field content. We define

$$
\tilde{H} \equiv H+i H^{\prime},
$$

with $H^{\prime}$ a second real antisymmetric representation of $\mathrm{SU}(4)$. The action of $\mathrm{U}(1)_{A}$ is

$$
\mathrm{U}(1)_{A}: \tilde{H} \rightarrow e^{i \theta} \tilde{H},
$$

where $\theta$ is the parameter of the $\mathrm{U}(1)_{A}$ transformation. The field $H^{\prime}$ introduces an additional $\mathrm{Sp}(4)$ singlet that is the analog of the $\eta^{\prime}$ in QCD and 5 additional scalars that form a multiplet of the $\mathrm{SO}(5)$ unbroken symmetry, and are the analogue of the $a_{0}$ isovectors of QCD. The treatment presented here is indeed a generalization of what done in the context of the linear-sigma-model description of low-energy QCD [34-38].

The presence of the anomaly produces a large mass for $\eta^{\prime}$. At high temperatures both the fermion condensate and the effect of the anomaly are suppressed. Hence, the mass splitting between $a_{0}$ and $\pi$ provides a measure of the level of breaking of $\mathrm{U}(1)_{A}$ in addition to global $\mathrm{SU}(4)$, and can be used to look for $\mathrm{SU}(4) \times \mathrm{U}(1)_{A}$ thermal restoration. Similar arguments hold in the case of QCD (see for example [39, 40] and references therein).

Because the $\sigma$ and $\eta^{\prime}$ are flavor singlets, and the flavor-singlet sector of the spectrum is more difficult to study numerically than the flavored channels, we will study the $a_{0}-\pi$ mass splitting in order to discuss the restoration of the axial $\mathrm{U}(1)_{A}$ at high temperatures. We will do so in the body of the paper, using numerical techniques based on the formulation of the theory on anisotropic lattices.

\subsection{Composite states: vectors}

The full set of spin-1 vector and axial-vector mesons spans the adjoint representation of the SU(4) global symmetry. A cartoon representing the EFT description of their longdistance dynamics is depicted in figure 1 , and represents a generalization of hidden local symmetry [41-46]. One extends the symmetry from $\mathrm{SU}(4)$ to $\mathrm{SU}(4)_{A} \times \mathrm{SU}(4)_{B}$, with $\mathrm{SU}(4)_{A}$ weakly gauged, with coupling $g_{\rho}$. Then one enlarges the field content to include two non-linear sigma-model fields $S$ and $\Sigma$. The non-linear sigma-model $S$ transforms as the bifundamental of $\mathrm{SU}(4)_{B} \times \mathrm{SU}(4)_{A}$, while the field $\Sigma$ transforms on the antisymmetric of $\mathrm{SU}(4)_{A}$ :

$$
S \rightarrow U_{B} S U_{A}^{\dagger}, \quad \Sigma \rightarrow U_{A} \Sigma U_{A}^{T} .
$$

In a composite-Higgs model, the $\mathrm{SM}$ gauge group $\mathrm{SU}(2)_{L} \times \mathrm{U}(1)_{Y}$ is a subgroup of $\mathrm{SU}(4)_{B}$.

The gauging of the $\mathrm{SU}(4)_{A}$ symmetry means that (for global $\mathrm{SU}(4)_{B}$ ) one has to introduce the covariant derivatives

$$
\begin{aligned}
& D_{\mu} S=\partial_{\mu} S-i g_{\rho} S A_{\mu}, \\
& D_{\mu} \Sigma=\partial_{\mu} \Sigma+i g_{\rho}\left(A_{\mu} \Sigma+\Sigma A_{\mu}^{T}\right),
\end{aligned}
$$




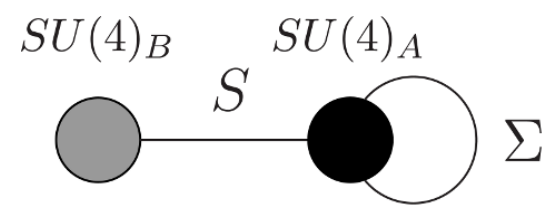

Figure 1. The moose diagram representing the EFT description of the vector mesons in the model.

\begin{tabular}{|c|c|c|c|}
\hline Label & Operator & Meson & $J^{P}$ \\
$S$ & $\overline{Q^{i}} Q^{j}$ & $a_{0}$ & $0^{+}$ \\
$P S$ & $\overline{Q^{i}} \gamma_{5} Q^{j}$ & $\pi$ & $0^{-}$ \\
$V$ & $\overline{Q^{i}} \gamma_{\mu} Q^{j}$ & $\rho$ & $1^{-}$ \\
$A V$ & $\overline{Q^{i}} \gamma_{5} \gamma_{\mu} Q^{j}$ & $a_{1}$ & $1^{+}$ \\
\hline
\end{tabular}

Table 2. Interpolating operators, and corresponding flavored particles (i.e. $i \neq j$ in the interpolating operators), studied in the body of the paper. Color and spinor indexes (summed over) are understood.

and then $\mathcal{L}_{0}$ is replaced by all possible 2-derivative invariant operators made by $S, \Sigma, D S$, $D \Sigma$, together with the kinetic term for the gauge bosons. Both $S$ and $\Sigma$ are non-vanishing in the vacuum, inducing the symmetry breaking pattern $\mathrm{SU}(4)_{A} \times \mathrm{SU}(4)_{B} \rightarrow \mathrm{Sp}(4)$, and all vectors are massive. $\langle\Sigma\rangle$ splits the mass of the $5 a_{1}$ and the $10 \rho$ mesons.

In unitary gauge, besides the heavy vectors only the physical pions are retained. They are linear combinations of the fluctuations of $S$ and $\Sigma$. The mass term for the pions is

$$
\mathcal{L}_{m}=-\frac{v^{3}}{4} \operatorname{Tr}\left\{M S \Sigma S^{T}\right\}+\text { h.c. }
$$

The quark masses also contribute to the masses of the spin-1 states in a more complicated way, that will be discussed elsewhere [47].

In the absence of the antisymmetric condensate (for $\langle\Sigma\rangle=0$ ), $\rho$ and $a_{1}$ mesons would be exactly degenerate. Their mass splitting is hence a measure of the amount of breaking $\mathrm{SU}(4) \rightarrow \mathrm{Sp}(4)$. In the main body of the paper we use the mass splitting between $\rho$ (vector) and $a_{1}$ (axial-vector) as a way to test whether the global symmetry is restored at high temperatures. The generalization to the case in which $\Sigma$ is replaced by $\tilde{H}$ does not require any new ingredients. In particular the restoration of the axial $\mathrm{U}(1)_{A}$ and of the global $\mathrm{SU}(4)$ can, at least in principle, be treated independently. We summarize in table 2 the properties of the states discussed in the body of the paper. One of the purposes of this paper is to make the first steps towards a quantitative assessment of the relation between the two phenomena at high temperature, in the specific theory of interest here.

\section{Numerical results: anisotropic lattice}

\subsection{Lattice action}

In this section, we describe the discretized Euclidean lattice action used for our numerical study. For the gauge sector, we modify the standard plaquette action by treating the oper- 
ators containing temporal gauge links separately from those solely containing spatial links,

$$
S_{g}[U]=\frac{\beta}{\xi_{g}^{0}}\left[\sum_{i}\left(\xi_{g}^{0}\right)^{2}\left(1-\frac{1}{N} \operatorname{Re} \operatorname{tr} \mathcal{P}_{0 i}\right)+\sum_{i<j}\left(1-\frac{1}{N} \operatorname{Re} \operatorname{tr} \mathcal{P}_{i j}\right)\right],
$$

where $\beta=2 N / g^{2}$ and $\xi_{g}^{0}$ are the lattice bare gauge coupling and the bare gauge anisotropy, respectively. The plaquette $\mathcal{P}$ is defined by

$$
\mathcal{P}_{\mu \nu}(x)=U_{\mu}(x) U_{\nu}(x+\hat{\mu}) U_{\mu}^{\dagger}(x+\hat{\nu}) U_{\nu}^{\dagger}(x),
$$

where $U_{\mu}(x)$ denotes the link variables. For the fermion sector, we use the Wilson action for fermions in the fundamental represention

$$
S_{f}[U, \bar{Q}, Q]=a_{s}^{3} a_{t} \sum_{x} \bar{Q}(x) D_{m} Q(x),
$$

with the massive Wilson-Dirac operator given by

$$
D_{m} Q(x)=m_{0} Q(x)+\frac{1}{2} \sum_{\mu} v_{\mu}\left[\gamma_{\mu}\left(\nabla_{\mu}+\nabla_{\mu}^{*}\right)-a_{\mu} \nabla_{\mu}^{*} \nabla_{\mu}\right] Q(x)
$$

where $\nabla$ and $\nabla^{*}$ denote the forward and backward covariant derivatives, respectively:

$$
\begin{aligned}
& \nabla_{\mu} Q(x)=\frac{1}{a_{\mu}}\left[U_{\mu}(x) Q(x+\hat{\mu})-Q(x)\right], \\
& \nabla_{\mu}^{*} Q(x)=\frac{1}{a_{\mu}}\left[Q(x)-U_{\mu}^{\dagger}(x-\hat{\mu}) Q(x-\hat{\mu})\right] .
\end{aligned}
$$

The ratio $v_{\mu}$ of the bare fermion to gauge anisotropy is introduced as it can be different to unity. From the redefinition of the fermion field $\left(Q \rightarrow \sqrt{v_{t}} Q\right.$ and $\left.m_{0} \rightarrow m_{0} / v_{t}\right)$, along with the introduction of the fermion anisotropy $\xi_{f}^{0}=\xi_{g}^{0} /\left(v_{s} / v_{t}\right)$, we rewrite eq. (3.4) as

$$
\begin{aligned}
D_{m} Q(x) \equiv & \left(D+m_{0}\right) Q(x)=\frac{1}{a_{t}}\left[\left(a_{t} m_{0}+1+\frac{3}{\xi_{f}^{0}}\right) Q(x)\right. \\
& -\frac{1}{2}\left(\left(1-\gamma_{0}\right) U_{0}(x) Q(x+\hat{0})+\left(1+\gamma_{0}\right) U_{0}^{\dagger}(x-\hat{0}) Q(x-\hat{0})\right) \\
& \left.-\frac{1}{2 \xi_{f}^{0}} \sum_{j}\left(\left(1-\gamma_{j}\right) U_{j}(x) Q(x+\hat{j})+\left(1+\gamma_{j}\right) U_{j}^{\dagger}(x-\hat{j}) Q(x-\hat{j})\right)\right] .
\end{aligned}
$$

For the rest of this paper we do not explicitly show the lattice spacings for convenience, i.e. $a_{t}=1$, except when we need to distinguish the spatial and temporal lattice spacings and to discuss the finite temperature.

The bare anisotropy parameters, $\xi_{g}^{0}$ and $\xi_{f}^{0}$, are renormalized such that physical probes at scales well below the cut-off $\sim 1 / a$ exhibit Euclidean symmetry, i.e. $\xi_{g}=\xi_{f}=\xi$. For the input quark mass, $M_{q}$, we parameterize the renormalized parameters $\left(\xi_{g}, \xi_{f}, M_{q}\right)$ as functions of bare parameters $\left(\xi_{g}^{0}, \xi_{f}^{0}, m_{0}\right)$. For a small region in the parameter space, we 


\begin{tabular}{|ccccccccc|}
\hline$m_{0}$ & $\xi_{g}^{0}$ & $\xi_{f}^{0}$ & $N_{\text {conf }}$ & $M_{\mathrm{PS}}$ & $M_{V}$ & $\xi_{g}$ & $\xi_{f}$ & $M_{\mathrm{PS}} / M_{V}$ \\
\hline-0.195 & 4.7 & 4.7 & 200 & $0.1659(8)$ & $0.1823(10)$ & $6.19(7)$ & $6.34(10)$ & $0.910(7)$ \\
-0.195 & 4.9 & 4.7 & 200 & $0.1544(6)$ & $0.1709(13)$ & $6.33(8)$ & $6.33(9)$ & $0.903(8)$ \\
-0.2 & 4.5 & 4.7 & 300 & $0.1616(5)$ & $0.1784(8)$ & $6.03(6)$ & $6.28(7)$ & $0.906(5)$ \\
-0.2 & 4.7 & 4.5 & 300 & $0.1743(5)$ & $0.1910(7)$ & $6.07(7)$ & $6.12(6)$ & $0.913(4)$ \\
-0.2 & 4.7 & 4.7 & 200 & $0.1504(6)$ & $0.1678(10)$ & $6.13(6)$ & $6.41(11)$ & $0.896(6)$ \\
-0.2 & 4.9 & 4.7 & 300 & $0.1399(5)$ & $0.1589(7)$ & $6.42(6)$ & $6.35(7)$ & $0.880(5)$ \\
-0.2 & 5.1 & 4.7 & 160 & $0.1279(13)$ & $0.1479(19)$ & $6.58(9)$ & $6.30(15)$ & $0.865(14)$ \\
-0.209 & 4.7 & 4.5 & 150 & $0.1455(7)$ & $0.1643(11)$ & $6.10(6)$ & $6.04(10)$ & $0.885(7)$ \\
-0.209 & 4.7 & 4.7 & 300 & $0.1169(7)$ & $0.1392(13)$ & $6.22(6)$ & $6.35(12)$ & $0.840(10)$ \\
-0.209 & 4.9 & 4.5 & 300 & $0.1336(6)$ & $0.1533(9)$ & $6.34(7)$ & $6.11(9)$ & $0.872(6)$ \\
-0.209 & 4.9 & 4.7 & 150 & $0.1023(9)$ & $0.1243(15)$ & $6.35(6)$ & $6.25(12)$ & $0.823(12)$ \\
$-0.215^{a}$ & 4.7 & 4.7 & 138 & $0.0904(21)$ & $0.118(5)$ & $6.04(9)$ &. & $0.77(3)$ \\
$-0.209^{b}$ & 4.7 & 4.7 & 300 & $0.1172(7)$ & $0.1382(11)$ & $6.13(6)$ & $6.42(13)$ &. \\
\hline
\end{tabular}

${ }^{a}$ : This ensemble is used only for the determination of $a_{i}$ and $c_{i}$ as the number of configurations is not large enough to determine $\xi_{f}$ in a reliable manner.

${ }^{b}$ : For this ensemble we carry out the measurements on the $128 \times 10^{3}$ lattice. Note that $M_{\mathrm{PS}} N_{s} \sim 7$. Compared to the $128 \times 12^{3}$ lattice, we find no significant differences in all measured quantities. As $M_{\mathrm{PS}} N_{s} \gtrsim 7$ for other ensembles, we therefore expect that the finite volume effects are negligible in the tuning of bare lattice parameters.

Table 3. Simulation parameters and results for the tuning of the lattice bare parameters of an anisotropic lattice. The masses of pseudoscalar (PS) and vector (V) mesons are measured in units of $a_{t}$.

assume that the renormalized parameters are linear in the bare parameters. We further assume that we are in the region of light quark masses, i.e. $M_{\mathrm{PS}}^{2} \sim M_{q}$, and arrive at the form [48]

$$
\begin{array}{r}
\xi_{g}\left(\xi_{g}^{0}, \xi_{f}^{0}, m_{0}\right)=a_{0}+a_{1} \xi_{g}^{0}+a_{2} \xi_{f}^{0}+a_{3} m_{0}, \\
\xi_{f}\left(\xi_{g}^{0}, \xi_{f}^{0}, m_{0}\right)=b_{0}+b_{1} \xi_{g}^{0}+b_{2} \xi_{f}^{0}+b_{3} m_{0}, \\
M_{\mathrm{PS}}^{2}\left(\xi_{g}^{0}, \xi_{f}^{0}, m_{0}\right)=c_{0}+c_{1} \xi_{g}^{0}+c_{2} \xi_{f}^{0}+c_{3} m_{0} .
\end{array}
$$

For each set of bare parameters, nonperturbative determinations of $\xi_{g}$ and $\xi_{f}$ are carried out through the interquark potential and the relativistic meson dispersion relation, respectively, which will be discussed in details in the following subsections.

\subsection{Simulation details}

We consider the lattice action in eq. (3.1) and eq. (3.3) with two mass-degenerate Wilson fermions. Configurations are generated using the Hybrid Monte Carlo(HMC) algorithms with the second order Omelyan integrator for Molecular Dynamics(MD) evolution, where different lengths of MD time steps $\delta \tau_{\mu}$ are used for gauge and fermion actions such that the acceptance rate is in the range of $75-85 \%$. The simulation codes are developed from the HiRep code [49] modified by implementing the gauge and fermion anisotropies described in section 3.1. To optimise the acceptance rate, we also treat the variance of temporal 
and spatial conjugate momenta differently by introducing a new tunable parameter [50], which is essentially equivalent to the multiscale anisotropic molecular dynamics update [48]. Without changing the validity of the algorithm, such a setup is helpful for the anisotropic lattice calculations through balancing the temporal and spatial MD forces: typically the former is larger than the latter approximately by the anisotropy in the lattice spacings.

Except the lattice of $N_{t} \times N_{s}^{3}=128 \times 10^{3}$ for the investigation of finite volume effects, all of the numerical calculations for the tuning of bare parameters are performed on $N_{t} \times N_{s}^{3}=128 \times 12^{3}$ lattices. We use periodic boundary conditions in each direction of both link variables and fermion fields. ${ }^{3}$ Twelve ensembles are created with different bare quark masses, gauge and fermion anisotropies at $\beta=2.0$, where the details are found in table 3 . Thermalization and autocorrelation times are estimated by monitoring the average plaquette expectation values. For each ensemble $N_{\text {conf }}=138-300$ configurations are accumulated after 200 trajectories for thermalization, where every two adjacent configurations are separated by one auto-correlation length of which the typical size is $8 \sim 12$ trajectories. The statistical errors for all quantities extracted in this work are obtained using the standard bootstrapping technique.

\subsection{Gauge anisotropy}

The gauge anisotropy $\xi_{g}$ is determined from the static potential using Klassen's method [51]. We first define the ratios of spatial-spatial and spatial-temporal Wilson loops by

$$
R_{s}(r, y)=\frac{W_{s s}(r, y)}{W_{s s}(r+1, y)} \text { and } R_{t}(r, t)=\frac{W_{s t}(r, t)}{W_{s t}(r+1, t)}
$$

respectively. In an asymptotic region, these ratios fall exponentially with the linear interquark potential and do not depend on $r, R_{s}(r, y) \sim e^{-a_{s} V_{s}\left(y a_{s}\right)}$ and $R_{t}(r, t) \sim e^{-a_{s} V_{s}\left(t a_{t}\right)}$. Finite volume effects are expected to be suppressed since they are canceled out in the ratios $[51,52]$. As the interquark potential at the same physical distance should yield the same value, one can extract the anisotropy $\xi_{g}$ by imposing $R_{s}(r, y) \equiv R_{t}\left(r, t=\xi_{g} y\right)$. In practice, we determine $\xi_{g}$ by minimizing [52]

$$
L\left(\xi_{g}\right)=\sum_{r, y} \ell\left(\xi_{g} ; r, y\right)
$$

with

$$
\ell\left(\xi_{g} ; r, y\right)=\frac{\left(R_{s}(r, y)-R_{t}\left(r, \xi_{g} y\right)\right)^{2}}{\left(\Delta R_{s}\right)^{2}+\left(\Delta R_{t}\right)^{2}},
$$

where $\Delta R_{s}$ and $\Delta R_{t}$ are the statistical errors of $R_{s}$ and $R_{t}$, respectively.

In the original Klassen's approach, the planar Wilson loops are considered where $r$ is either $x$ or $z$. A typical difficulty in this approach is the limited number of data points as one quickly encounters a severe signal-to-noise problem in the calculations of the large Wilson loops. By noting that $\vec{r}$ can be any two-dimensional path in the $x$-z plane with $r=\sqrt{x^{2}+z^{2}}$, we extend the Klassen's method by including nonplanar Wilson loops along

\footnotetext{
${ }^{3}$ We have checked that using antipeoriodic boundary conditions in the time direction for fermions give compatible results as expected in zero-temperature calculations.
} 


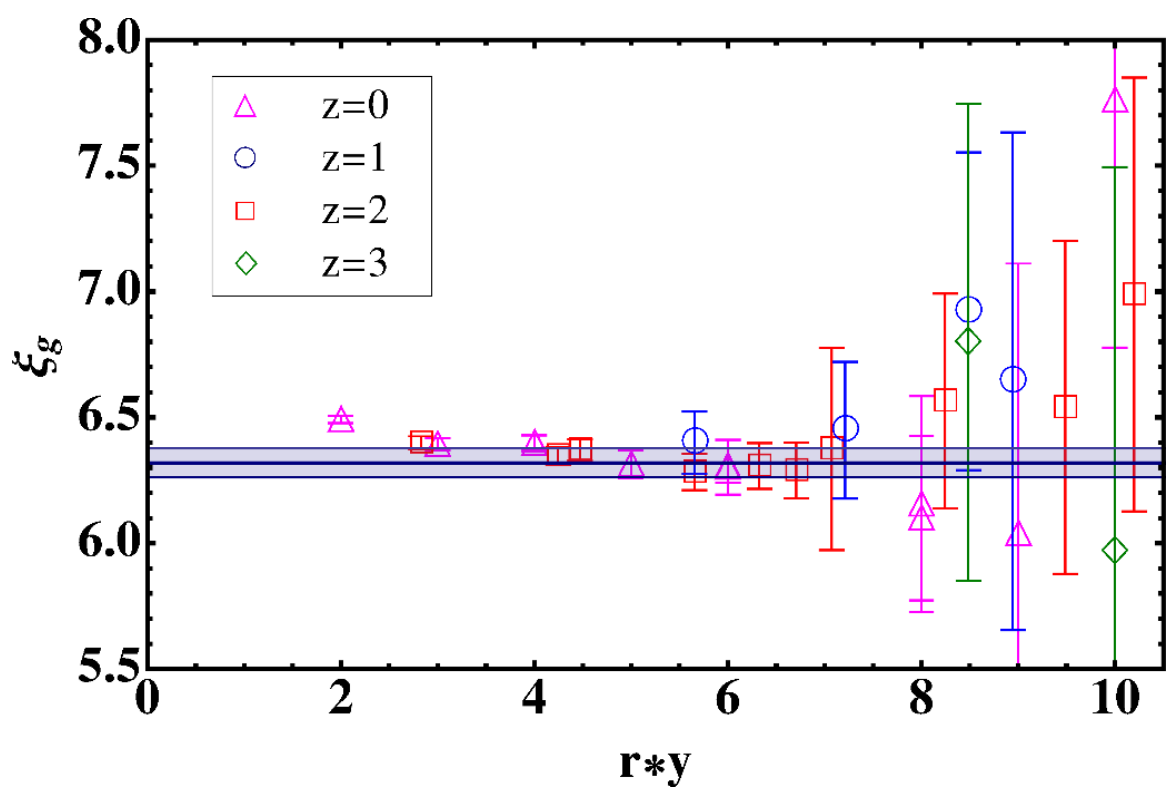

Figure 2. Lattice artefacts due to nonplanar Wilson loops. Different colored points denote the $\xi_{g}$ obtained by using eq. (3.10) with $z=0, \cdots, 3$ for a given $r * y$, while the blue band denotes the extracted value of $\xi_{g}$. The details are found in the main text.

the closed path $C_{y}(x, z, y)$ and $C_{t}(x, z, t)$ with $x \geq z$. To maximize the overlap with the physical ground state the shortest paths in the $x$-z plane are considered using the Bresenham algorithm which has been applied for the lattice study of quark antiquark potential, i.e. see [53]. Analogous to the planar case, we define $\vec{r}=(x, z)$ and $\vec{r}+1=$ $(x+1, z)$ for a fixed value of $z$.

Using the generalized Klassen's method, we are able to secure enough data points having reasonable statistical errors. As a consequence, not only do we find the clean signal of an asymptotic region in which $\xi_{g}$ converges, but also reduce the statistical error of the gauge anisotropy $\xi_{g}$. However, due to the breaking of rotational symmetry on the lattice, results obtained mixing on-axis and off-axis loops might be affected by a large systematics. To investigate this issue, we calculate $\xi_{g}$ by minimizing the function $\ell\left(\xi_{g} ; r, y\right)$ with $z=0, \cdots, 3$, corresponding to the different shapes of the 2-dimensional paths. The results are shown in figure 2 . We find no significant deviations between colored data, suggesting that any potential effect of the breaking of rotational symmetry cancels in the ratios of Wilson loops in eq. (3.8). For $r * y \geq 5$ all data points are statistically consistent with one another. The measured value of $\xi_{g}$ is denoted by the blue band in the figure, where its extraction is discussed in the following.

In figure 3 we plot $\xi_{g}$, obtained by using eq. (3.9), as a function of $\min (r * y)$ for four different sets of data: all planar Wilson loops (purple triangle), planar Wilson loops except $y=1$ (blue circle), planar and nonplanar Wilson loops except $y=1$ (red square), and planar and nonplanar Wilson loops except $y=1$ and $r=1$ (green diamond). The largest value of $r * y$ is the one before we encounter significant numerical noise. 


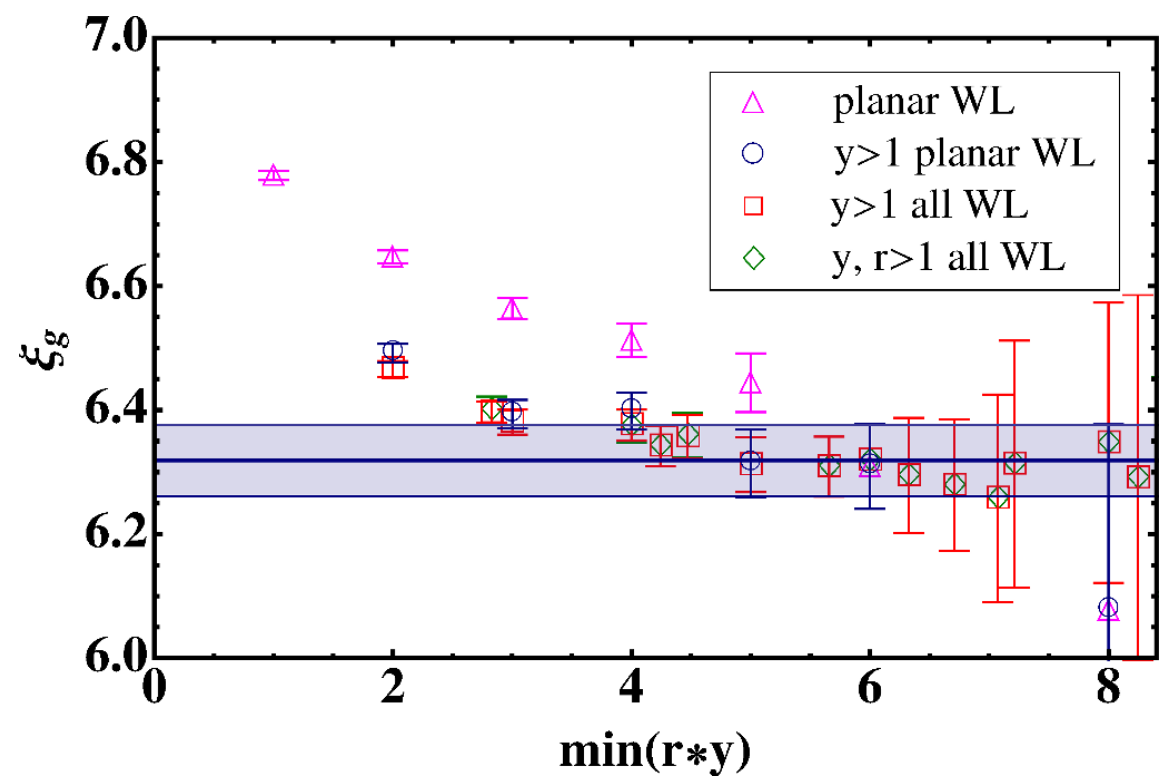

Figure 3. Gauge anisotropy $\xi_{g}$ extracted from the ratios of Wilson loops. Different colored points denote the values of $\xi_{g}$ obtained by using eq. (3.9) with different sets of data for a given $\min (r * y)$, while the blue band denotes the extracted value of $\xi_{g}$. Details are found in the main body of the paper.

For all ensembles we find that $\xi_{g}$ converges to the asymptotic value at around $\min (r *$ $y)=4 \sim 6$ and thus we choose $\min (r * y)=6$, as for this value we expect the size of systematic errors to be small compared to the statistical error. Since the inclusion of $y=1$ Wilson loops causes significant systematic effects due to short-range lattice artefacts, as can be seen in the plots (see also the discussion in [48, 52], in the case of QCD), we exclude these Wilson loops for the determination of $\xi_{g}$. In summary, we calculate the asymptotic value of $\xi_{g}$ using planar and nonplanar Wilson loops, except the ones having $y=1$, at $\min (r * y)=6$ and the results are reported in table 3 .

\subsection{Fermion anisotropy}

The fermion anisotropy $\xi_{f}$ is determined through the leading-order relativistic dispersion relation of mesons

$$
E^{2}\left(p^{2}\right)=m^{2}+\frac{p^{2}}{\xi_{f}^{2}}, \quad \vec{p}=2 \pi \vec{n} / N_{s},
$$

where $N_{s}$ is the spatial lattice size. The energy $E$ and the mass $m$ are in units of $a_{t}$, while the momentum $\vec{p}$ is in units of $a_{s}$. In the Euclidean formulation, meson two-point correlation functions exponentially fall off with the lowest energy at an asymptotically large time. In practice, it is useful to define an effective mass,

$$
m_{\mathrm{eff}}(t)=\cosh ^{-1}\left(\frac{C(t+1)+C(t-1)}{2 C(t)}\right),
$$

where $C(t)$ is the ensemble average of meson correlators. Then, ground state energies are obtained from a constant fit to the plateau of $m_{\mathrm{eff}}$ in the asymptotic region of large $t$. In the 

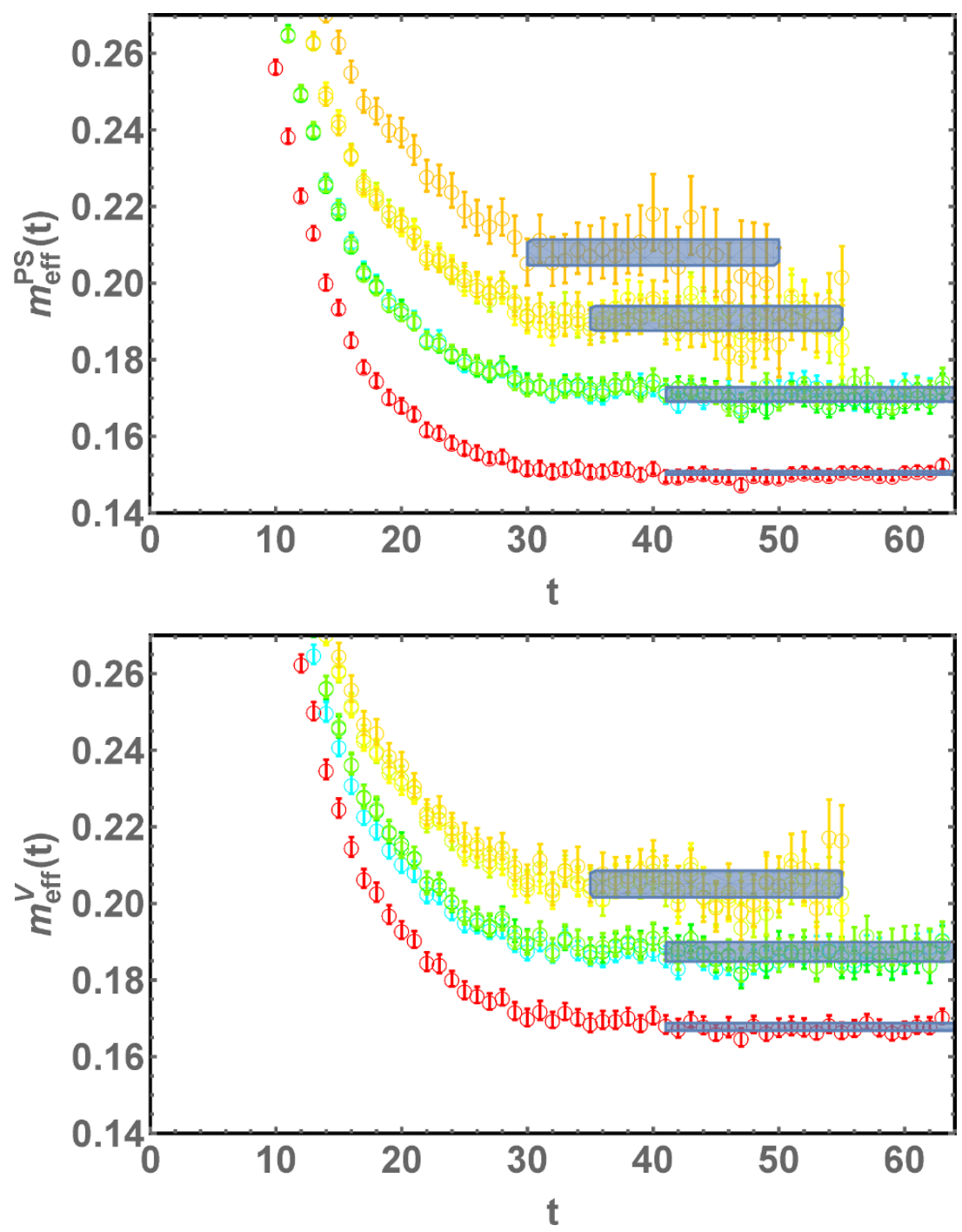

Figure 4. Effective mass plots for a pseudoscalar meson $m_{\mathrm{eff}}^{\mathrm{PS}}$ and a vector meson $m_{\mathrm{eff}}^{V}$. Red, green, yellow, brown colors represent different momenta $|\vec{n}|=0,1,2,3$, respectively. The blue bands denote the ground state energies obtained by fitting the effective mass to a constant in the asymptotic region. The lattice bare parameters used in these plots are $\beta=2.0, m_{0}=-0.2$, $\xi_{g}^{0}=4.7, \xi_{f}^{0}=4.7$, and $N_{t} \times N_{s}^{3}=128 \times 12^{3}$.

case of zero momentum these energies are nothing but the meson masses. The measured masses of pseudoscalar and vector mesons are reported in table 3.

As an example, in figure 4 we show the effective mass plots for pseudoscalar and vector mesons with $m_{0}=0.2, \xi_{g}^{0}=\xi_{f}^{0}=4.7$ and $\beta=2.0$. We construct the meson interpolating operators at source and sink using point sources. Various momentum projections with $|\vec{n}|=0,1,2,3$ are denoted by red, green, yellow and brown colors, respectively, while the measured ground state energies are denoted by the blue bands.

In figure 5 we plot the resulting squared energy $E^{2}$ as a function of $|\vec{n}|^{2}$ and find a good linearity, consistent with eq. (3.11). In the determination of $\xi_{f}$, to minimize the systematic 

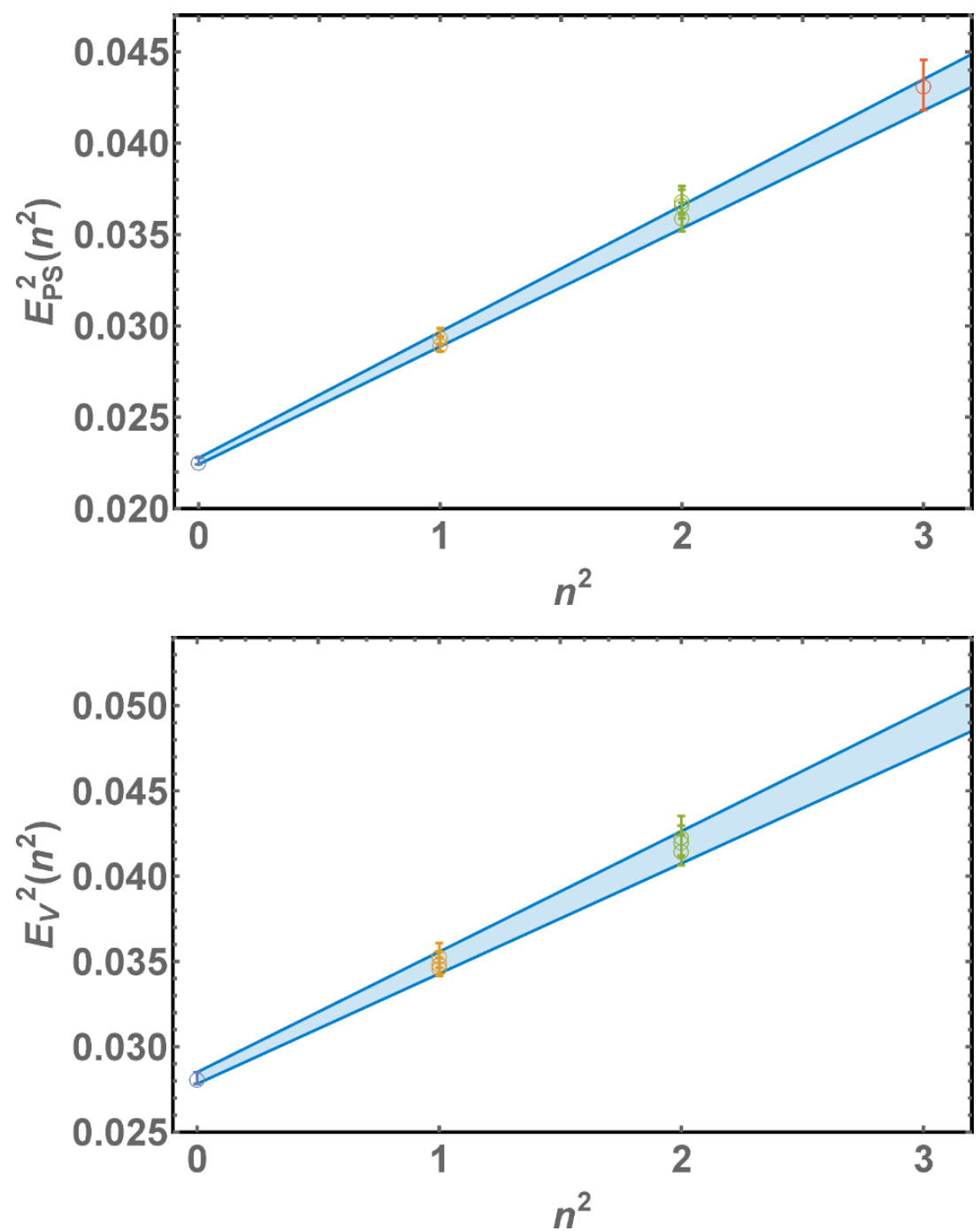

Figure 5. Squared energy as a function of squared momentum $|\vec{n}|^{2}$ for a pseudoscalar meson $E_{\mathrm{PS}}^{2}$ and a vector meson $E_{V}^{2}$. The blue band is obtained by fitting data over $n^{2}=[0,1]$ to a linear function of eq. (3.11). The lattice bare parameters used in these plots are $\beta=2.0, m_{0}=-0.2$, $\xi_{g}^{0}=4.7, \xi_{f}^{0}=4.7$, and $N_{t} \times N_{s}^{3}=128 \times 12^{3}$.

effects due to excited state contamination at higher momenta, we only use the lowest four momentum vectors $\vec{n}=(0,0,0),(1,0,0),(0,1,0),(0,0,1)$ in the linear fit of $E^{2}\left(|\vec{n}|^{2}\right)$ to eq. (3.11). As seen in the figures, the fit results denoted by blue bands explain the data very well. The extracted value of $\xi_{f}=6.41(11)$ from a pseudoscalar meson is in good agreement with the one from a vector meson, $\xi_{f}=6.36(14)$, and shows better precision. Therefore, for the tuning of lattice bare parameters we use $\xi_{f}$ from pseudoscalar mesons which are summarized in table 3. 


\subsection{Tuning results}

To determine the coefficients, $a_{i}, b_{i}$, and $c_{i}$, we perform the simultaneous $\chi^{2}$ fit of the numerical data in table 3 to the functions in eq. (3.7). The results are

$$
\begin{array}{llll}
a_{0}=0.6(16), & a_{1}=0.97(13), & a_{2}=0.31(23), & a_{3}=2(4), \\
b_{0}=1.8(24), & b_{1}=0.06(18), & b_{2}=1.1(3), & b_{3}=4(7), \\
c_{0}=0.475(5), & c_{1}=-0.0168(4), & c_{2}=-0.0375(6), & c_{3}=0.986(11),
\end{array}
$$

where the values of $\chi^{2}$ per degrees of freedom are $1.72,0.72,0.23$, respectively. In appendix $\mathrm{C}$ we show some examples of the results of the fit in the two-dimensional spaces of the renormalized and bare parameters.

Our interpretation of the above results requires that we comment on a few important features. First of all, renormalized anisotropies are somewhat larger than the bare anisotropies, which we interpret as a signal of the fact that the calculations are performed far from the weak coupling limit. Secondly, we find that the coefficients $a_{2}$ and $b_{1}$ are small, in particular, $b_{1}$ is zero within the statistical errors. In the quenched approximation, one would expect that the gauge and fermion anisotropies can be determined independently. The mild dependences of $\xi_{f}$ on $\xi_{g}^{0}$ and $\xi_{g}$ on $\xi_{f}^{0}$ are consistent with the fact that this part of the numerical study is performed in the regime of heavy quarks. Yet, we note that over the range of considered lattice parameters our results show a good linear dependence of the squared mass of a pseudoscalar meson $M_{\mathrm{PS}}^{2}$ on the bare quark mass $m_{0}$, which is consistent with our use of eq. (3.7) to extrapolate to the limit of vanishing physical mass for the quarks.

In order to determine the values of the bare parameters at our chosen reference point we impose the following renormalization conditions:

$$
\xi_{g}\left(\xi_{g}^{0 *}, \xi_{f}^{0 *}, m_{0}^{*}\right)=\xi_{f}\left(\xi_{g}^{0 *}, \xi_{f}^{0 *}, m_{0}^{*}\right)=\xi, \quad M_{\mathrm{PS}}^{2}\left(\xi_{g}^{0 *}, \xi_{f}^{0 *}, m_{0}^{*}\right)=m_{p s}^{2} .
$$

Solving eq. (3.7) with our target renormalized parameters of $\xi=6.3$ and $m_{p s}^{2}=0.005$, we find

$$
\xi_{g}^{0 *}=4.84(8), \xi_{f}^{0 *}=4.72(12), m_{0}^{*}=-0.2148(37) .
$$

We will use these choices for the lattice parameters in measuring the physical properties of the field theory. Note that $m_{0}^{*}$ falls slightly outside the range of masses used in this part of the study (see table 3), and hence we expect some (small) residual quark mass and symmetry-breaking effects to be present in our physical simulations.

\section{Numerical results: finite temperature}

From now on, the lattice bare parameters are fixed by the central values in eq. (3.15) along with $\beta=2.0$. We perform finite temperature calculations on the anisotropic lattices of $N_{t} \times 16^{3}$ and $N_{t} \times 16^{2} \times 24$. Simulation details and numerical results for these two lattices are summarized in tables 4,5 and 6 . Two different values of $N_{z}$ are considered to estimate the systematic errors due to excited state contaminations in the calculations of screening 


\begin{tabular}{|cccccccc|}
\hline$N_{z}$ & $N_{t}$ & $T / T_{c}$ & $N_{\text {conf }}$ & $N_{z}$ & $N_{t}$ & $T / T_{c}$ & $N_{\text {conf }}$ \\
\hline 16 & 16 & 2.44 & 200 & 24 & 8 & 4.88 & 200 \\
& 20 & 1.95 & 200 & & 12 & 3.25 & 200 \\
& 24 & 1.63 & 200 & & 16 & 2.44 & 225 \\
& 28 & 1.39 & 200 & & 20 & 1.95 & 150 \\
& 30 & 1.30 & 200 & & 24 & 1.63 & 200 \\
& 36 & 1.08 & 200 & & 28 & 1.39 & 250 \\
& 40 & 0.98 & 200 & & 36 & 1.08 & 380 \\
& 128 & 0.30 & 215 & & 42 & 0.93 & 388 \\
& & & & 48 & 0.81 & 390 \\
& & & & 56 & 0.70 & 337 \\
\hline
\end{tabular}

Table 4. Details of the ensembles of the $N_{t} \times 16^{3}$ and $N_{t} \times 16^{2} \times 24$ lattices. All calculations use $\xi_{g}^{0 *}=4.84, \xi_{f}^{0 *}=4.72, m_{0}^{*}=-0.2148$ and $\beta=2.0$.

\begin{tabular}{|ccccccc|}
\hline$T / T_{c}$ & $M_{\mathrm{PS}}^{S}$ & $M_{S}^{S}$ & $M_{V}^{S}$ & $M_{A V}^{S}$ & $R_{S}$ & $R_{V}$ \\
\hline 2.44 & $0.3322(2)$ & $0.3823(8)$ & $0.3475(2)$ & $0.3879(10)$ & $0.070(1)$ & $0.0549(13)$ \\
1.95 & $0.2815(6)$ & $0.3292(14)$ & $0.3045(5)$ & $0.340(2)$ & $0.078(2)$ & $0.055(3)$ \\
1.63 & $0.2355(6)$ & $0.281(2)$ & $0.2629(7)$ & $0.2938(18)$ & $0.088(4)$ & $0.056(3)$ \\
1.39 & $0.1937(13)$ & $0.236(5)$ & $0.2272(12)$ & $0.248(4)$ & $0.099(10)$ & $0.045(8)$ \\
1.30 & $0.1783(13)$ & $0.228(5)$ & $0.2110(13)$ & $0.235(5)$ & $0.123(11)$ & $0.054(10)$ \\
1.08 & $0.1312(11)$ & $0.201(6)$ & $0.1699(15)$ & $0.191(5)$ & $0.210(13)$ & $0.057(13)$ \\
0.98 & $0.1147(12)$ & $0.180(4)$ & $0.1525(16)$ & $0.185(6)$ & $0.222(12)$ & $0.096(16)$ \\
0.30 & $0.0758(3)$ & $0.200(8)$ & $0.1068(11)$ & $0.213(9)$ & $0.449(16)$ & $0.331(19)$ \\
\hline
\end{tabular}

Table 5. Simulation results for the $N_{t} \times 16^{3}$ lattice. To compensate the anisotropy of the Euclidean lattice, meson screening masses $M^{S}$ are obtained from the measured masses divided by $\xi=6.3$. All calculations use $\xi_{g}^{0 *}=4.84, \xi_{f}^{0 *}=4.72, m_{0}^{*}=-0.2148$ and $\beta=2.0$.

\begin{tabular}{|ccccccc|}
\hline$T / T_{c}$ & $M_{\mathrm{PS}}^{S}$ & $M_{S}^{S}$ & $M_{V}^{S}$ & $M_{A V}^{S}$ & $R_{S}$ & $R_{V}$ \\
\hline 4.88 & $0.4724(2)$ & $0.5021(8)$ & $0.4770(2)$ & $0.5044(7)$ & $0.0305(9)$ & $0.0279(8)$ \\
3.25 & $0.3885(2)$ & $0.4293(15)$ & $0.3975(3)$ & $0.432(2)$ & $0.0498(18)$ & $0.041(2)$ \\
2.44 & $0.33257(17)$ & $0.3833(8)$ & $0.34760(18)$ & $0.3905(5)$ & $0.0709(11)$ & $0.0581(7)$ \\
1.95 & $0.2813(5)$ & $0.3268(15)$ & $0.3043(4)$ & $0.3371(14)$ & $0.075(3)$ & $0.051(2)$ \\
1.63 & $0.2326(7)$ & $0.275(2)$ & $0.2617(7)$ & $0.290(3)$ & $0.083(4)$ & $0.052(5)$ \\
1.39 & $0.1909(9)$ & $0.234(4)$ & $0.2239(9)$ & $0.251(3)$ & $0.102(10)$ & $0.058(6)$ \\
1.08 & $0.1295(9)$ & $0.202(7)$ & $0.1680(11)$ & $0.194(4)$ & $0.218(17)$ & $0.072(11)$ \\
0.93 & $0.1036(6)$ & $0.181(4)$ & $0.1440(11)$ & $0.170(5)$ & $0.272(10)$ & $0.081(13)$ \\
0.81 & $0.0880(5)$ & $0.191(6)$ & $0.1254(9)$ & $0.186(5)$ & $0.369(15)$ & $0.193(12)$ \\
0.70 & $0.0798(4)$ & $0.183(7)$ & $0.1107(13)$ & $0.199(6)$ & $0.393(17)$ & $0.284(16)$ \\
\hline
\end{tabular}

Table 6. Simulation results for a $N_{t} \times 16^{2} \times 24$ lattice. To compensate the anisotropy of the Euclidean lattice, meson screening masses $M^{S}$ are obtained from the measured masses divided by $\xi=6.3$. All calculations use $\xi_{g}^{0 *}=4.84, \xi_{f}^{0 *}=4.72, m_{0}^{*}=-0.2148$ and $\beta=2.0$. 
masses. The algorithms for the generation of gauge ensembles have been discussed in section 3.2 .

Before we discuss the numerical results of finite temperature calculations in details, we perform a zero temperature calculation in order to check how well the tuned bare parameters are working. Using the ensemble of $128 \times 16^{3}$ in table 5 , we obtain $\xi_{g}=6.29(4)$, $\xi_{f}=6.1(2)$, and $M_{p s}^{2}=0.00517(14)$. These results are compatible with the renormalized parameters of $\xi=6.3$ and $m_{p s}^{2}=0.005$, where the largest uncertainty occurs in the detemination of $\xi_{f}$ with $\sim 3 \%$. Finite volume effects are expected to be negligible as the lattice volume is much larger than the size of the pseudo-scalar meson, $m_{p s} L \sim 7$.

Adopting anti-periodic boundary condition along the temporal direction, temperature is defined by $T \equiv \frac{1}{N_{t} a_{t}}$. We will find it convenient to measure the temperature in units of the (pseudo-)critical temperature $T_{c}$, discussed and measured in the next section.

\subsection{Deconfinement crossover}

As is the case for QCD with small number of quarks, our model is also expected to exhibit confinement at low temperature and form a quark-gluon plasma across the (pseudo-)critical temperature $T_{c}$. Although the Polyakov loop is not an exact order parameter when the number of quarks is finite, it is widely used as an indicator of deconfinement. Following the method used in $[54,55]$, we define the expectation value of the renormalized Polyakov loop ${ }^{4}$ by

$$
L_{R}(T)=Z_{L}^{N_{t}} L_{0}(T)
$$

where the bare Polyakov loop $L_{0}(T)$ is related to the bare free energy $F_{0}(T)$ as $L_{0}(T)=$ $\exp \left(-F_{0}(T)\right)$. The multiplicative renormaliztion constant is defined by $Z_{L}=\exp \left(-\Delta F_{0}\right)$, which only captures the short distant physics and thus is independent on the temperature. As different choices of $Z_{L}$ denote different renormalization schemes, to incorporate the scheme dependence on the detemination of $T_{c}$ we impose a renormalization condition for a given temperature $T_{R}$ by $L_{R}\left(T_{R}\right) \equiv$ constant.

We consider three renormalization schemes, defined by the conditions $L_{R}\left(N_{t}=24\right)=$ $0.9, L_{R}\left(N_{t}=24\right)=0.5$, and $L_{R}\left(N_{t}=20\right)=0.9$ respectively. The results are shown in figure 6 . The temperature $T_{c}$ is determined from the peak of the susceptibility of the Polyakov loop, $\chi\left(L_{R}\right)=\partial L_{R} / \partial T$, denoted by dashed lines in the figure. Combining the statistical uncertainty and the systematic uncertainty of scheme dependences in quadrature, we find that $T_{c} a_{t}=0.0255(25)$, or equivalently that $N_{t}^{c}=39(4)$. As anticipated, we will measure temperatures in units of this $T_{c}$ in the following.

\subsection{Temporal correlation functions}

At zero temperature, the Euclidean two-point correlation functions of mesonic observables fall off with a single exponential at a large time so that the ground state energy of mesons can be extracted in a clear way in principle. In the finite temperature lattice calculations

\footnotetext{
${ }^{4}$ For the discussion of the renormalized Polaykov loop and its scheme dependence, we refer the reader to $[56,57]$.
} 


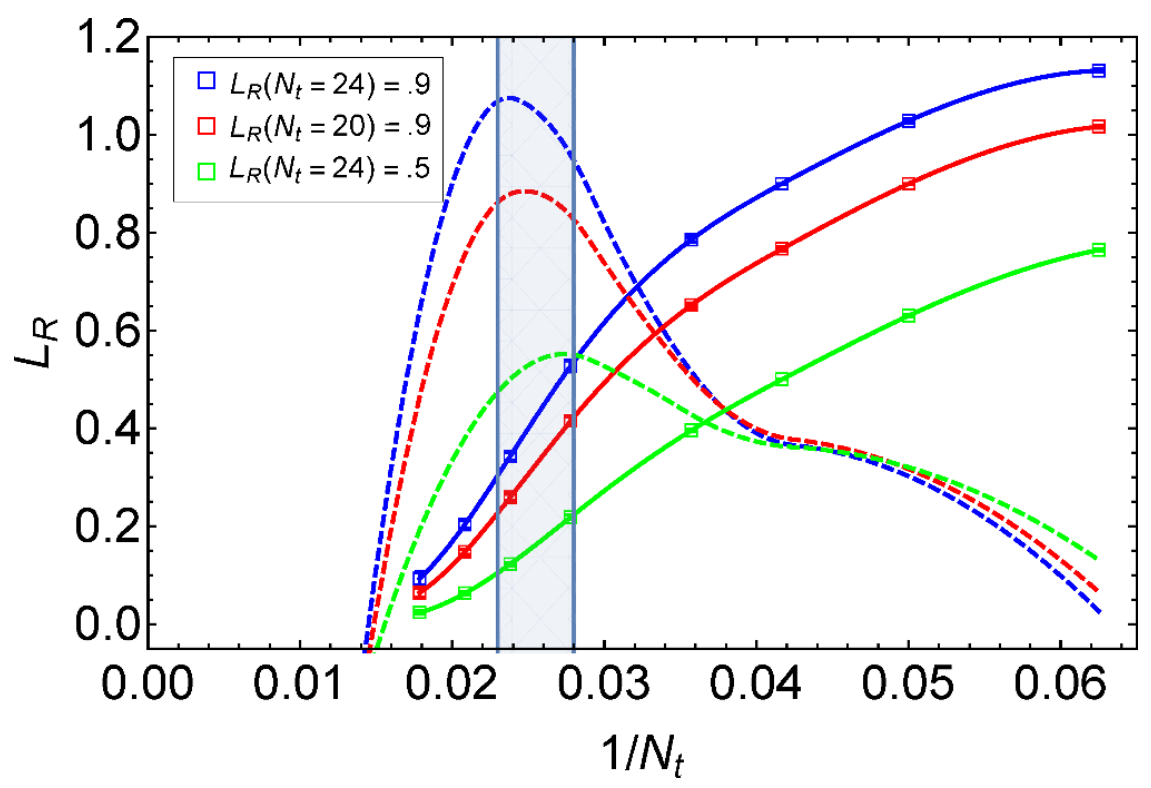

Figure 6. Renormalized Polyakov loop and their susceptibility. The renormalized Polyakov loops $L_{R}$ denoted by empty squares are obtained from the ensembles of $N_{t} \times 16^{2} \times 24$ with $N_{t}$ ranged over $[16,56]$. The solid curves are the interpolation of $L_{R}$ connected by cubic splines, while the dashed curves are the corresponding susceptibility $\chi\left(L_{R}\right)$, the derivatives of $L_{R}$ with $N_{t}^{-1}$. Different colors are associated with different renormalization conditions, while the blue band denotes the (pseudo)critical temperature $T_{c}$ with uncertainties as described in the text.

this process is affected by some limitations. Firstly, the maximum available physical temporal extent is limited by the inverse of the temperature. In addition, a single exponential analysis becomes subtle as the spectral function of mesons no longer exhibits a sharp peak at the mass of mesons. In this case, it is more desirable to investigate the correlation functions by themselves.

We introduce the normalized correlation function with the reference choice $t=N_{t} / 2$ :

$$
C_{N_{t} / 2}(t)=\frac{C(t)}{C\left(N_{t} / 2\right)}
$$

We consider isovector pseudo-scalar, scalar, vector, and axial-vector mesons, where the corresponding interpolating fields are defined by

$$
\begin{aligned}
\mathcal{O}_{\mathrm{PS}}(x) & =\bar{Q}(x) \gamma_{5} Q(x), & \mathcal{O}_{S}(x) & =\bar{Q}(x) Q(x), \\
\mathcal{O}_{V}^{i}(x) & =\bar{Q}(x) \gamma^{i} Q(x), & \mathcal{O}_{A V}^{i}(x) & =\bar{Q}(x) \gamma_{5} \gamma^{i} Q(x),
\end{aligned}
$$

respectively (flavour indices selecting non-singlet states are understood). In order to improve the statistics, we use stochastic wall sources [58] for the study of meson spectrum at finite temperature. Using these mesonic operators we compute the function $C_{N_{t} / 2}(t)$. In figure 7 we show the results of $\log C_{N_{t} / 2}(t)$ for $N_{t}=48$ and 40, which exemplify the typical behaviors of $C_{N_{t} / 2}(t)$ below and near $T_{c}$ respectively.

By comparing the two plots in figure 7 one can see that while at low temperature $\left(N_{t}=\right.$ 48) the vector and axial-vector correlators are different, they become hard to distinguish 

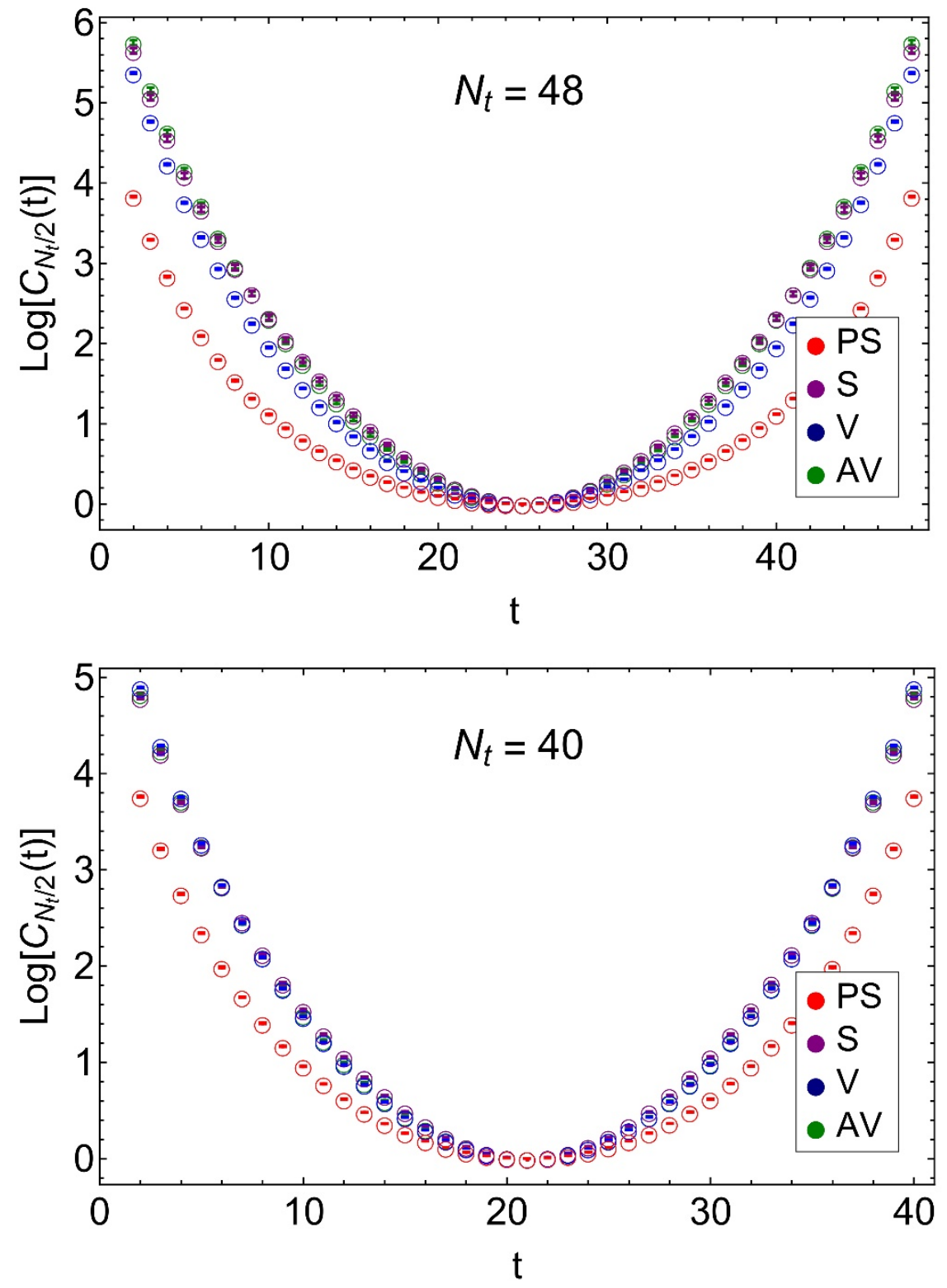

Figure 7. Temporal correlation functions for pseudoscalar(red), scalar(purple), vector(blue), and axial vector(green) mesons. For a given Euclidean time $t$, we plot the logarithms of the correlation functions normalized by the correlation functions at $N_{t} / 2$.

from one another in proximity of $T_{c}\left(N_{t}=40\right)$. The overlap of $C_{N_{t} / 2}(t)$ between vector and axial-vector mesons can be considered as an indication of the parity doubling in the vector channel and thus the restoration of the global SU(4) symmetry. By contrast, the situation for scalar and pseudo-scalar correlators is quite different, as we will discuss better by looking at spatial correlation functions in the next subsection, and indicates that at this temperature we do not yet see evidence of the restoration of the $\mathrm{U}(1)_{A}$ symmetry. Notice that the correlation functions still satisfy the Weingarten's mass inequalities [59]. 


\subsection{Spatial correlation functions}

In contrast to the temporal correlation function, the spatial correlation function at finite temperature exhibits a single exponential decay at large time. The decay rate is called screening mass, as it defines the effective length scale associated with the excitation of mesonic operators in the medium [60]. At zero temperature the screening mass is equivalent to the meson mass, as the temporal and spatial correlation functions share the same spectral function.

By using the meson interpolating fields in eq. (4.3), we calculate the ensemble average of spatial correlators $C(z)$ along the $z$-direction and extract the masses in units of $a_{s}$ using the analysis method described in section 3.4. Notice that in our anisotropic lattice calculations the spatial and temporal lengths are measured differently. To have the consistent lattice unit of mass in $a_{t}$, we therefore define the screening mass $M^{S}$ by multiplying $\xi^{-1}$ to the measured spatial masses. In addition to the screening masses, we define the following normalized mass ratios

$$
R_{V}(T)=\frac{M_{A V}^{S}(T)-M_{V}^{S}(T)}{M_{A V}^{S}(T)+M_{V}^{S}(T)}
$$

for the vector channel, and

$$
R_{S}(T)=\frac{M_{S}^{S}(T)-M_{\mathrm{PS}}^{S}(T)}{M_{S}^{S}(T)+M_{\mathrm{PS}}^{S}(T)}
$$

for the scalar channel. These quantities are useful to quantify the level of parity doubling in the mass spectrum.

Our main results are presented in table 5 and 6 , as well as in figure 8 and 9 . The error bar of each data point only represents the statistical uncertainty. We show explicitly the comparison between $N_{t} \times 16^{3}$ (black) and $N_{t} \times 16^{2} \times 24$ (red) lattices. The level of agreement of the two ensembles implies that there is no significant systematic uncertainty due to excited state contaminations.

By looking first at the vector and axial-vector masses, we see a plateau in $R_{V}$ above $T_{c}$, which together with the change of behavior of the masses above $T_{c}$ strongly suggests that parity partners are degenerate and the global symmetry is effectively restored. There is small deviation from zero in the mass ratio at asymptotically large values of $T$, that may be the result of finite spacing, finite mass and possibly other small lattice artefacts.

In the case of the scalar channel, the plateau in $R_{S}$ appears at somewhat larger temperature, $\sim 1.5 T_{c}$. This result may imply that the axial $\mathrm{U}(1)_{A}$ and global $\mathrm{SU}(4)$ symmetries are restored at different temperatures. However, this is not conclusive, for several reasons. First of all, because we do not know what kind of transition is appearing in the underlying dynamical model, and it is likely that $T_{c}$ actually identifies a cross-over. But also because we do not know how much each of the lattice artefacts affects the results, and it might be that different observables are affected in different amounts by the finite quark mass, or the finite value of the coupling. A relevant discussion in the context of two-flavor QCD can be found in [39], for instance, where the numerical results strongly suggest that the symmetry restorations occur simultaneously in the massless limit. 

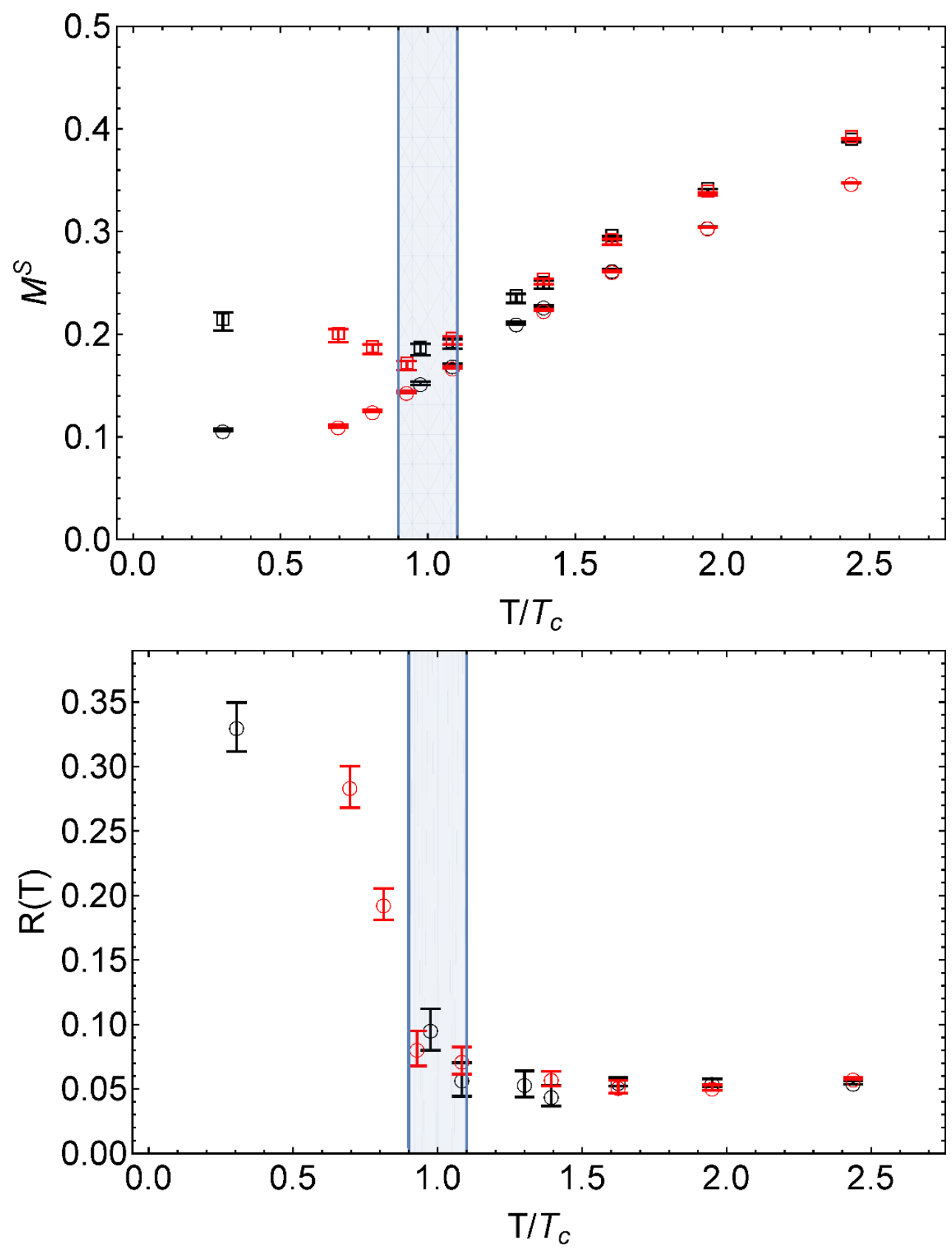

Figure 8. Screening masses of vectors (circles) and axial vector (squares) mesons (top panel) and the corresponding mass ratio defined in eq. (4.4) (bottom panel). Black and red points are extracted from ensembles with $N_{t} \times 16^{3}$ and $N_{t} \times 16^{2} \times 24$ lattice points, respectively. The temperature is expressed in units of $T_{c}$. The blue vertical band denotes the uncertainty of $T_{c}$ itself. 

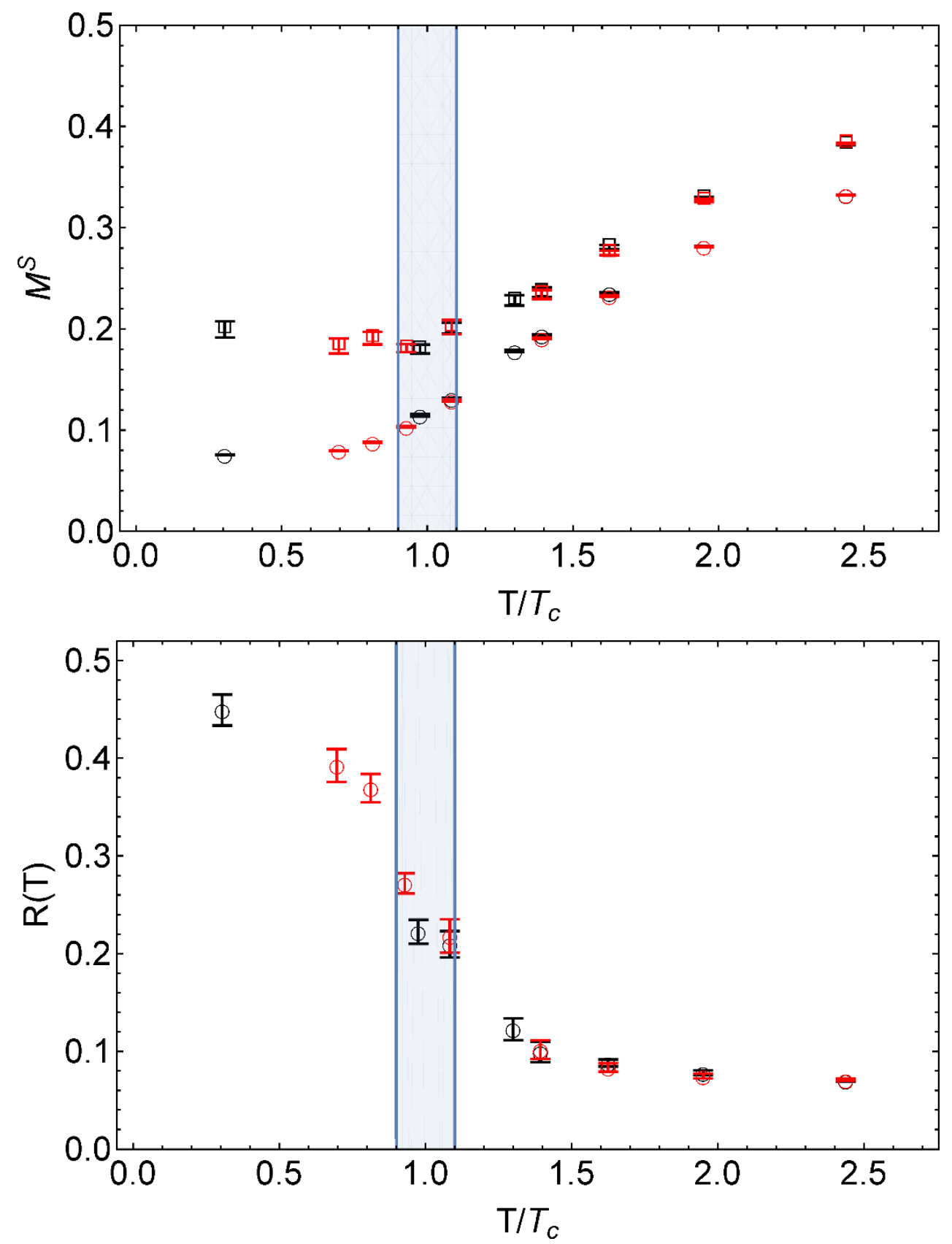

Figure 9. Screening masses of pseudoscalar (circles) and scalar (squares) mesons (top panel) and the corresponding mass ratio defined in eq. (4.5) (bottom panel). Black and red points are extracted from ensembles with $N_{t} \times 16^{3}$ and $N_{t} \times 16^{2} \times 24$ lattice points, respectively. The temperature is expressed in units of $T_{c}$. The blue vertical band denotes the uncertainty of $T_{c}$ itself. 

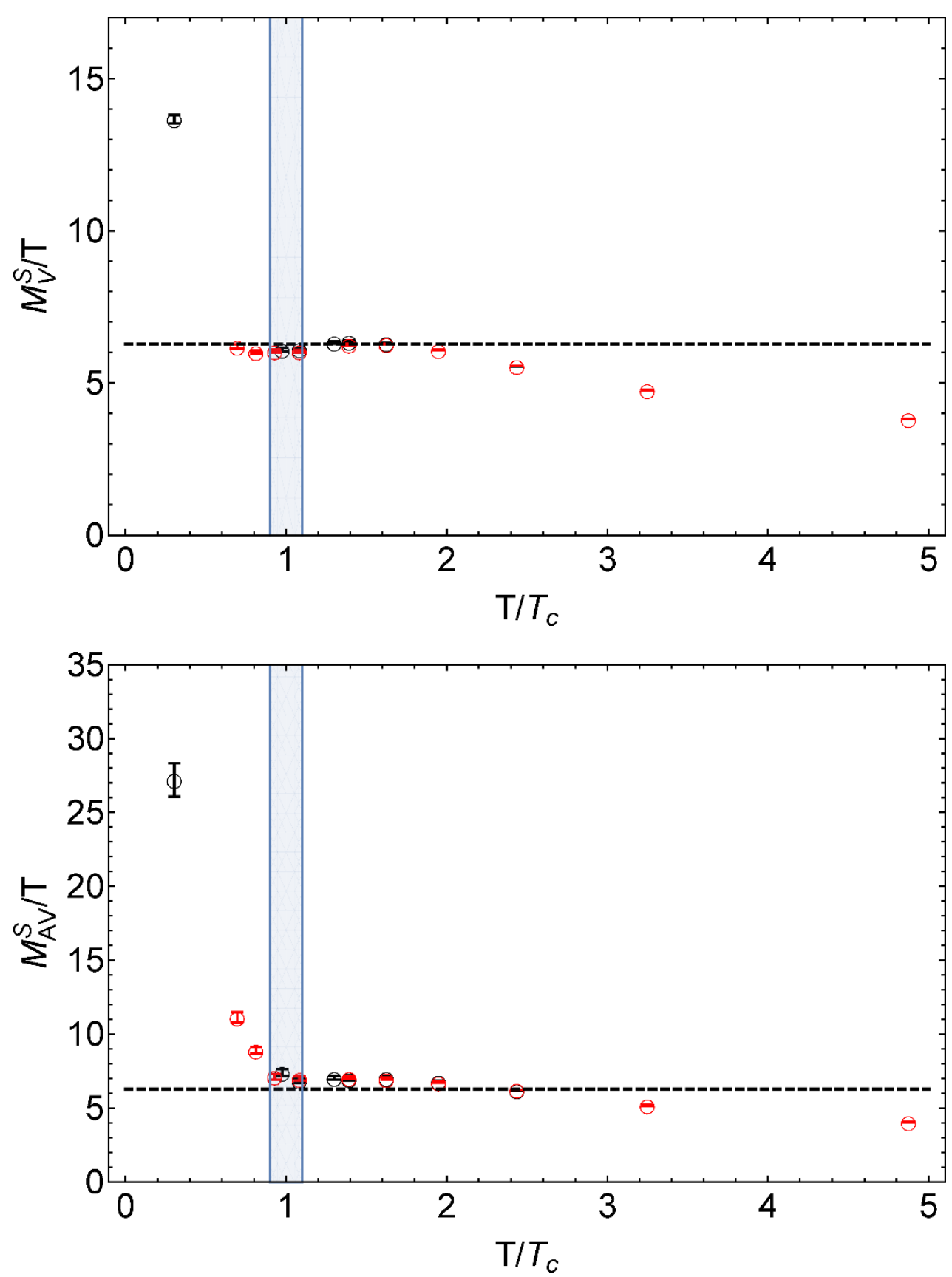

Figure 10. Screening masses normalized by temperature for vector (top) and axial vector (bottom) mesons. Black and red points are extracted from ensembles of $N_{t} \times 16^{3}$ and $N_{t} \times 16^{2} \times 24$, respectively. The temperature is in units of $T_{c}$, where the blue vertical band denotes the uncertainty of $T_{c}$ itself. 

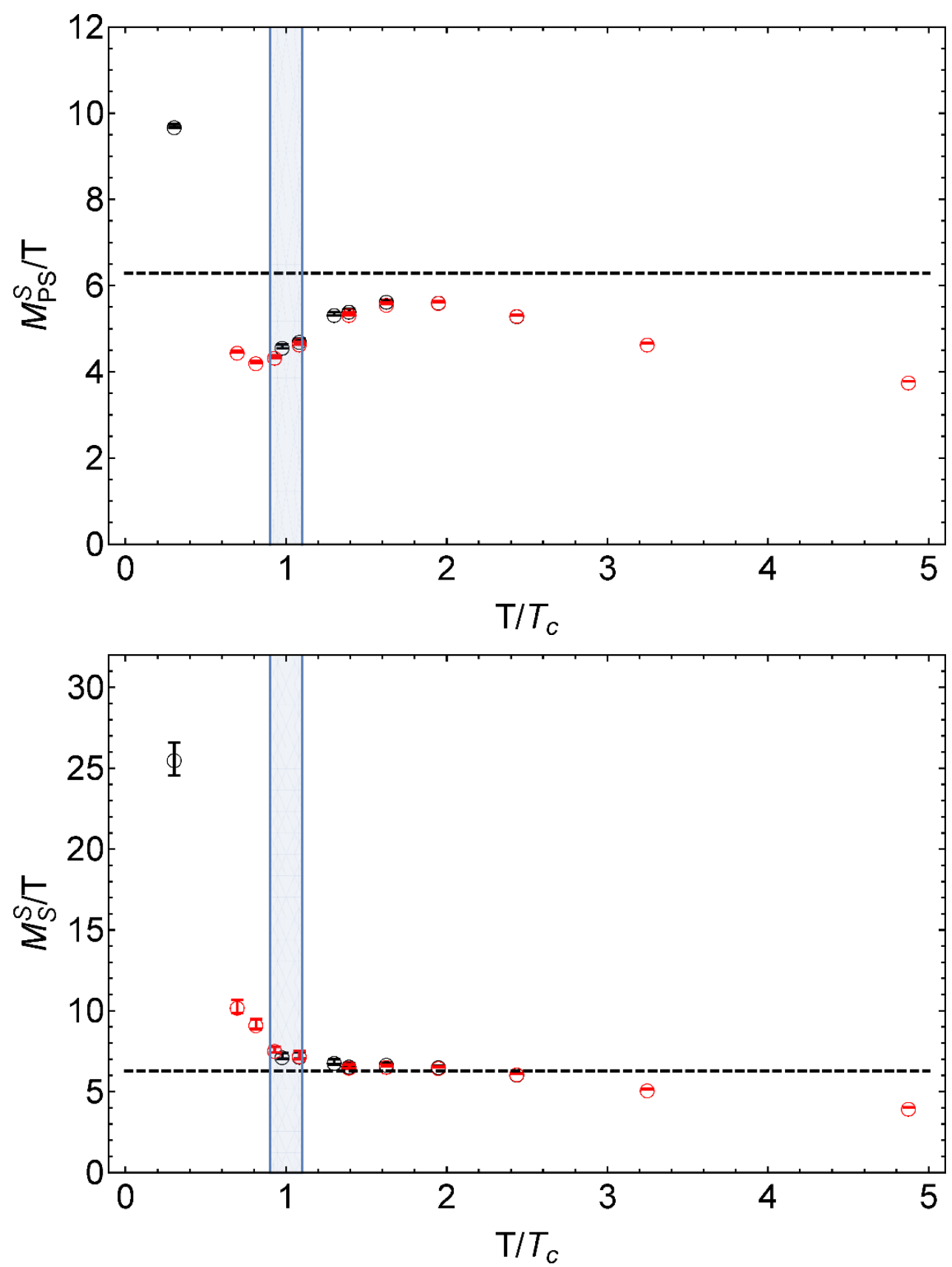

Figure 11. Screening masses normalized by temperature for pseudoscalar (top) and scalar (bottom) mesons. Black and red points are extracted from ensembles of $N_{t} \times 16^{3}$ and $N_{t} \times 16^{2} \times 24$, respectively. The temperature is in units of $T_{c}$, where the blue vertical band denotes the uncertainty of $T_{c}$. 
In the finite temperature calculations, it is often suggested to plot the screening mass divided by the temperature as it shows linear dependency above $T_{c}$. The results are shown in figure 10 and figure 11. The black dashed line corresponds to $2 \pi$ which is associated with the Matsubara frequency for massless free quarks. For all mesonic channels, data points approach the dashed line as the temperature increases and seem to form a plateau. However, they start to deviate from the plateau above $2 T_{c}$, possibly as a consequence of the finite lattice spacing. ${ }^{5}$ This suggests that in looking at $R_{V}$ and $R_{S}$ (and in general in discussing parity-doubling) one should not include in the physical very high temperatures, but rather restrict attention to $T \lesssim 2 T_{c}$.

\section{Discussion}

We collected numerical evidence of the fact that the high-temperature behavior of the $\mathrm{SU}(2)$ theory with $N_{f}=2$ Dirac fundamental fermions differs in three respects from the lowtemperature one. The numerical study of the Polyakov loop and its fit shows the existence of a pronounced peak in the susceptibility. Its position identifies a temperature $T_{c}$, that we interpret in terms of the deconfinement (cross-over) temperature. While the study of the details of the transition would require a dedicated program, this result is accurate enough to allow us to clearly separate the high- $T$ and low- $T$ regimes, and concentrate on the symmetry properties of the physical spectrum above $T_{c}$.

The study of temporal correlation functions shows that for $T>T_{c}$ the vector and axial-vector 2-point functions have compatible $t$-dependence, supporting the hypothesis that parity doubling is emerging at $T_{c}$, and global symmetry is restored. This is confirmed by the study of spatial correlation functions, in which one clearly sees that the behavior of the screening masses of vectors and axial-vector mesons changes at $T \sim T_{c}$ : while the two masses are different and depend on $T$ in two different ways when $T<T_{c}$, for $T>T_{c}$ the masses come close to one another, and, most importantly, show the same $T$-dependence.

This last observation suggests that the small splitting in the masses we observe is due to a combination of lattice artefacts (in particular finite spacing and finite quark mass). To confirm or disprove this statement, one would need to extend the study in this paper, and consider more than one value of the bare coupling and of the bare quark mass, in order to extrapolate them both to the physically relevant regime. By doing so, one might not only be able to show that the mass difference between vectors and axial-vectors vanishes, but also to study other properties of the transition itself, such as its order.

The numerical study of the scalar and pseudo-scalar masses, in which we focused on cleaner states that form a fundamental of $\mathrm{SO}(5)$, shows qualitative features that are in broad agreement with the restoration also of the axial $\mathrm{U}(1)_{A}$ symmetry at high temperature. Our data on spatial correlation functions seems to suggest that this is taking place at a larger temperature $T^{\prime} \sim 1.5 T_{c}$. This is also supported by the fact that in the temporal correlation functions we do not see the effect of parity doubling in the spin-0 correlators, for the same choice $N_{t}=40$ for which the vector and axial-vector correlators do agree with one another. This is the most striking element of novelty of this study, although it must be considered as preliminary.

\footnotetext{
${ }^{5}$ As shown in $2+1$ Lattice QCD calculations using staggered fermions [31], the size of these lattice artefacts can significantly be reduced if highly improved lattice fermions being used.
} 
This paper is to be understood as a first step in what is a potentially broad and extensive research program. The results obtained are in good agreement with what expected on field-theory grounds about the non-trivial behavior of this theory at high temperatures: it deconfines, and both the global $\mathrm{SU}(4)$ and axial $\mathrm{U}(1)_{A}$ symmetries are restored. Two main sets of explorations are interesting to pursue in the future. On the one side, it is interesting to perform precision studies of this system, in which larger statistics, and a broader set of values of the lattice parameters, are used in order to establish whether the three transitions we identified are distinct (and in this case how to classify them, and precisely measure the critical temperatures), or whether they are just three manifestations of the broader phenomenology related to a cross-over.

On the other hand, it is also interesting to understand how the system reacts to the introduction of additional sources of symmetry breaking at the Lagrangian level. For example, the weak gauging of a subgroup of $\mathrm{Sp}(4)$ (as in phenomenological compositeHiggs models), is going to break the global symmetry of the model, and with it the large degeneracies of states. It would be useful to know how these phenomena depend on finite temperature. Closely related, although possibly simpler, is the question of what happens at finite $\mu$ : given that $\mathrm{SU}(2)$ is pseudo-real, this model is free of the traditional sign problem of similar models with larger gauge groups. It should hence be possible to attempt a more general study of the phase diagram as a function of both $T$ and $\mu$.

The richness of the field theory behavior of this model, the wide variety of its possible applications and the fact that this study shows that its thermal features are amenable to quantitative numerical studies, all contribute to making it an ideal environment in which to study highly non-trivial phenomena, which might shed light on many aspects of direct relevance to QCD, TC and composite-Higgs scenarios. In this paper we performed a first study along these lines, mainly aimed at collecting evidence of symmetry restoration at high temperature. We also discussed ways to improve our results, and suggested avenues for further investigation, which we will pursue in the future.

\section{Acknowledgments}

This work is supported in part by the STFC Consolidated Grant ST/L000369/1. The work of J.-W. L. is additionally supported by Korea Research Fellowship program funded by the Ministry of Science, ICT and Future Planning through the National Research Foundation of Korea (2016HID3A1909283). The authors thank S. Hands, G. Aarts, B. Jager, F. Attanasio and E. Bennett for discussions. Numerical computations were executed in part on the HPC Wales systems, supported by the ERDF through the WEFO (part of the Welsh Government).

\section{A Spinors and global symmetries}

We summarise in this appendix some useful notation about spinors, and show explicitly the origin of the enhanced global SU(4) symmetry of the model.

The space-time metric is $\eta_{\mu \nu} \equiv \operatorname{diag}\{1,-1,-1,-1\}=\eta^{\mu \nu}$, and the Dirac algebra is defined by the relation $\left\{\gamma^{\mu}, \gamma^{\nu}\right\}=2 \eta^{\mu \nu}$, with $\gamma^{0}$ hermitian and $\gamma^{i}$ anti-hermitian, such that 
$\gamma^{0} \gamma^{\mu} \gamma^{0}=\gamma^{\mu \dagger}$. Chirality is related to $\gamma_{5} \equiv i \gamma^{0} \gamma^{1} \gamma^{2} \gamma^{3}$, the left-handed(LH) chiral projector is $P_{L}=\frac{1}{2}\left(\mathbb{I}_{4}+\gamma_{5}\right)$, and a 4 -components LH chiral spinor $Q_{L}$ obeys $P_{L} Q_{L}=Q_{L}$. The charge-conjugation matrix $C=i \gamma^{2} \gamma^{0}$ obeys $C \gamma_{\mu} C^{-1}=-\gamma^{\mu T}$ and $C^{2}=-\mathbb{I}_{4}=-C C^{\dagger}$.

The chiral representation for the gamma matrices, in terms of the Pauli matrices $\tau^{i}$, is

$$
\gamma^{0}=\left(\begin{array}{cc}
0 & \mathbb{I}_{2} \\
\mathbb{I}_{2} & 0
\end{array}\right), \quad \gamma^{i}=\left(\begin{array}{cc}
0 & -\tau^{i} \\
\tau^{i} & 0
\end{array}\right), \quad \gamma^{5}=\left(\begin{array}{cc}
\mathbb{I}_{2} & 0 \\
0 & -\mathbb{I}_{2}
\end{array}\right), \quad C=\left(\begin{array}{cc}
-i \tau^{2} & 0 \\
0 & i \tau^{2}
\end{array}\right) \text {. }
$$

The following is immediate:

$$
\gamma^{0} \gamma^{\mu}=\left(\begin{array}{cc}
\bar{\sigma}^{\mu} & 0 \\
0 & \sigma^{\mu}
\end{array}\right), \quad C \gamma^{0} \gamma^{\mu} C^{-1}=\left(\begin{array}{cc}
\sigma^{\mu} & 0 \\
0 & \bar{\sigma}^{\mu}
\end{array}\right)
$$

where $\sigma^{\mu}=\left(1,-\tau^{i}\right)$ and $\bar{\sigma}^{\mu}=\left(1, \tau^{i}\right)$.

A Majorana spinor $\lambda$ obeys $\lambda= \pm \lambda_{C} \equiv \pm C \bar{\lambda}^{T}= \pm C \gamma^{0} \lambda^{*}= \pm i \gamma^{2} \lambda^{*}$. We resolve the \pm ambiguity by conventionally choosing the + sign .

Given a 2-component spinor $u$ we can build a 4-component Majorana spinor as

$$
\lambda=\left(\begin{array}{c}
u \\
i \tau^{2} u^{*} \equiv-\tilde{C} u^{*}
\end{array}\right)
$$

so that $\lambda=\lambda_{C}=\lambda_{L}+\lambda_{R}$, where

$$
\lambda_{L}=\left(\begin{array}{l}
u \\
0
\end{array}\right), \quad \lambda_{R}=\left(\begin{array}{c}
0 \\
i \tau^{2} u^{*} \equiv-\tilde{C} u^{*}
\end{array}\right) .
$$

In 4-component notation this ensures that $\lambda_{L}=C{\overline{\lambda_{R}}}^{T}$ and $\bar{\lambda}_{L}=\lambda_{R}^{T} C=-\lambda_{R}^{T} C^{-1}$.

With these definitions in place, and making use of the fact that Grassmann variables anticommute, after some algebra one finds that

$$
i \overline{\lambda_{R}} \gamma^{\mu} \partial_{\mu} \lambda_{R}=i \overline{\lambda_{L}} \gamma^{\mu} \partial_{\mu} \lambda_{L}+\frac{1}{2} \partial_{\mu}\left(-i \overline{\lambda_{L}} \gamma^{\mu} \lambda_{L}+i \overline{\lambda_{R}} \gamma^{\mu} \lambda_{R}\right)
$$

which implies that the kinetic term can be written equivalently in terms of $\lambda_{R}$ as of $\lambda_{L}$ (the total derivative can be dropped), or equivalently one can write it in terms of the 4-component Majorana spinor $\lambda$ (with an overall factor of $\frac{1}{2}$ to avoid double counting).

We specify now the model of interest in this paper, with $\mathrm{SU}(2)$ gauge symmetry. Starting from the 2-component spinors $q^{i a}$, with $i=1, \cdots, 4$ the flavor index and $a=1,2$ the color index, we can build four LH and four right-handed(RH) 4-component spinors as

$$
q_{L}^{j a}=\left(\begin{array}{c}
q^{j a} \\
0
\end{array}\right), \quad q_{R}^{j a}=\epsilon^{a b}\left(\begin{array}{c}
0 \\
\left(-\tilde{C} q^{j *}\right)_{b}
\end{array}\right),
$$

with $j=1, \cdots, 4$. Notice that the charge-conjugation used for the $\mathrm{RH}$ spinors implies to lower the $\mathrm{SU}(2)$ indexes, as it turns a fundamental of $\mathrm{SU}(2)$ in its conjugate. The essential property of $\mathrm{SU}(2)$ is that this can be compensated by the $\epsilon^{a b}$ antisymmetric tensor. 
One can define two Dirac spinors $Q^{i a}=q_{L}^{i a}+q_{R}^{i+2 a}$, with $i=1,2$. We identify such $Q^{i a}$ with the Dirac spinors that form the fundamental matter fields of the $\mathrm{SU}(2)$ gauge theory. Because of the structure of the gamma matrices, the kinetic terms do not couple different chiralities, and hence we can write

$$
\mathcal{L}_{K}=\sum_{i=1,2} i \overline{Q^{i}}{ }_{a} \gamma^{\mu}\left(D_{\mu} Q^{i}\right)^{a}=\sum_{i=1,2}\left(i \overline{q_{L}^{i}{ }_{a}} \gamma^{\mu}\left(D_{\mu} q_{L}^{i}\right)^{a}+i \overline{q_{R}^{i+2}}{ }_{a} \gamma^{\mu}\left(D_{\mu} q_{R}^{i+2}\right)^{a}\right)
$$

which makes it immediately visible that there is a $\mathrm{U}\left(N_{f}\right)^{t} \times \mathrm{U}\left(N_{f}\right)^{t}=\mathrm{U}(1)_{A} \times \mathrm{U}(1)_{B}^{t} \times$ $\mathrm{SU}(2)_{L}^{t} \times \mathrm{SU}(2)_{R}^{t}$ global symmetry, as would be true in any $\mathrm{SU}\left(N_{c}\right)$ gauge theory.

For $\mathrm{SU}(2)$ the global symmetry is actually larger: by making use of four LH 4component spinors and of eq. (A.5) one has

$$
i \overline{q_{R a}^{i}} \gamma^{\mu}\left(D_{\mu} q_{R}^{i}\right)^{a}=i \overline{q_{L a}^{i}} \gamma^{\mu}\left(D_{\mu} q_{L}^{i}\right)^{a}
$$

and hence we can write

$$
\mathcal{L}_{K}=\sum_{i=1}^{4} i \overline{q_{L a}^{i}} \gamma^{\mu}\left(D_{\mu} q_{L}^{i}\right)^{a}=\sum_{i=1}^{4} i\left(q^{i \dagger}\right)_{a} \bar{\sigma}^{\mu}\left(D_{\mu} q^{i}\right)^{a},
$$

which is manifestly $\mathrm{SU}(4) \times \mathrm{U}(1)_{A}$ invariant. Notice that besides the $\mathrm{SU}(2)^{t} \times \mathrm{SU}(2)^{t}$, the $\mathrm{SU}(4) \sim \mathrm{SO}(6)$ group includes also the $\mathrm{U}(1)_{B}^{t}$ associated with baryon number.

For completeness, we can explicitly verify that

$$
\mathcal{L}_{m}=-m \overline{Q^{i}}{ }_{a} Q^{i a}=-m \epsilon_{a b} q^{i+2 a} \tilde{C} q^{i b}+\text { h.c. }=-m \frac{1}{2} \epsilon_{a b} q^{n a T} \tilde{C} q^{m b} \Omega_{n m}+\text { h.c. },
$$

where the fact that $\Omega$ is antisymmetric comes from the antisymmetric $\epsilon_{a b}$. This is a Majorana mass, with $M=m \Omega$, which breaks explicitly the symmetry to $\operatorname{Sp}(4)$. The $\mathrm{U}(1)_{B}^{t}$ is a subgroup of $\mathrm{Sp}(4) \sim \mathrm{SO}(5)$, hence the spectrum of composite states cannot be classified in terms of baryon number, as mesons and baryons are in common $\mathrm{Sp}(4)$ multiplets. In the case one gauges the baryon number, then the symmetry would be explicitly broken back to the familiar $\mathrm{U}(2)^{2}$.

\section{B SU(4) and $\mathrm{Sp}(4)$ algebra}

The $N^{2}-1=15$ generators of $\mathrm{SU}(4)$ are hermitean traceless $4 \times 4$ complex matrices $T^{A}$. The $\operatorname{Sp}(4)$ subgroup is defined as the matrices that leave invariant the symplectic $\Omega$, which we write as

$$
\Omega=\left(\begin{array}{cccc}
0 & 0 & 1 & 0 \\
0 & 0 & 0 & 1 \\
-1 & 0 & 0 & 0 \\
0 & -1 & 0 & 0
\end{array}\right)
$$

$\mathrm{Sp}(4)$ is generated by the subset of 10 generators of $\mathrm{SU}(4)$ that obey the relation

$$
\Omega T^{A}+T^{A T} \Omega=0, \quad \text { for } A=6, \cdots 15
$$


while the 5 broken generators obey

$$
\Omega T^{A}-T^{A T} \Omega=0, \quad \text { for } A=1, \cdots 5 .
$$

By imposing the normalization $\operatorname{Tr} T^{A} T^{B}=\frac{1}{2} \delta^{A B}$, we write the 15 matrices as follows.

$$
\begin{aligned}
& T^{1}=\frac{1}{2 \sqrt{2}}\left(\begin{array}{cccc}
0 & 1 & 0 & 0 \\
1 & 0 & 0 & 0 \\
0 & 0 & 0 & 1 \\
0 & 0 & 1 & 0
\end{array}\right), \quad T^{2}=\frac{1}{2 \sqrt{2}}\left(\begin{array}{cccc}
0 & -i & 0 & 0 \\
i & 0 & 0 & 0 \\
0 & 0 & 0 & i \\
0 & 0 & -i & 0
\end{array}\right), \quad T^{3}=\frac{1}{2 \sqrt{2}}\left(\begin{array}{cccc}
1 & 0 & 0 & 0 \\
0 & -1 & 0 & 0 \\
0 & 0 & 1 & 0 \\
0 & 0 & 0 & -1
\end{array}\right), \\
& T^{4}=\frac{1}{2 \sqrt{2}}\left(\begin{array}{cccc}
0 & 0 & 0 & -i \\
0 & 0 & i & 0 \\
0 & -i & 0 & 0 \\
i & 0 & 0 & 0
\end{array}\right), \quad T^{5}=\frac{1}{2 \sqrt{2}}\left(\begin{array}{cccc}
0 & 0 & 0 & 1 \\
0 & 0 & -1 & 0 \\
0 & -1 & 0 & 0 \\
1 & 0 & 0 & 0
\end{array}\right), \quad T^{6}=\frac{1}{2 \sqrt{2}}\left(\begin{array}{cccc}
0 & 0 & -i & 0 \\
0 & 0 & 0 & -i \\
i & 0 & 0 & 0 \\
0 & i & 0 & 0
\end{array}\right), \\
& T^{7}=\frac{1}{2 \sqrt{2}}\left(\begin{array}{cccc}
0 & 0 & 0 & -i \\
0 & 0 & -i & 0 \\
0 & i & 0 & 0 \\
i & 0 & 0 & 0
\end{array}\right), \quad T^{8}=\frac{1}{2 \sqrt{2}}\left(\begin{array}{cccc}
0 & -i & 0 & 0 \\
i & 0 & 0 & 0 \\
0 & 0 & 0 & -i \\
0 & 0 & i & 0
\end{array}\right), \quad T^{9}=\frac{1}{2 \sqrt{2}}\left(\begin{array}{cccc}
0 & 0 & -i & 0 \\
0 & 0 & 0 & i \\
i & 0 & 0 & 0 \\
0 & -i & 0 & 0
\end{array}\right) \text {, } \\
& T^{10}=\frac{1}{2}\left(\begin{array}{llll}
0 & 0 & 1 & 0 \\
0 & 0 & 0 & 0 \\
1 & 0 & 0 & 0 \\
0 & 0 & 0 & 0
\end{array}\right), \quad T^{11}=\frac{1}{2 \sqrt{2}}\left(\begin{array}{llll}
0 & 0 & 0 & 1 \\
0 & 0 & 1 & 0 \\
0 & 1 & 0 & 0 \\
1 & 0 & 0 & 0
\end{array}\right), \quad T^{12}=\frac{1}{2}\left(\begin{array}{llll}
0 & 0 & 0 & 0 \\
0 & 0 & 0 & 1 \\
0 & 0 & 0 & 0 \\
0 & 1 & 0 & 0
\end{array}\right) \text {, } \\
& T^{13}=\frac{1}{2 \sqrt{2}}\left(\begin{array}{cccc}
0 & 1 & 0 & 0 \\
1 & 0 & 0 & 0 \\
0 & 0 & 0 & -1 \\
0 & 0 & -1 & 0
\end{array}\right), \quad T^{14}=\frac{1}{2 \sqrt{2}}\left(\begin{array}{cccc}
1 & 0 & 0 & 0 \\
0 & -1 & 0 & 0 \\
0 & 0 & -1 & 0 \\
0 & 0 & 0 & 1
\end{array}\right), \quad T^{15}=\frac{1}{2 \sqrt{2}}\left(\begin{array}{cccc}
1 & 0 & 0 & 0 \\
0 & 1 & 0 & 0 \\
0 & 0 & -1 & 0 \\
0 & 0 & 0 & -1
\end{array}\right) \text {. }
\end{aligned}
$$

The 5 Goldstone bosons can be written as $\pi(x)=\sum_{A=1}^{5} \pi^{A}(x) T^{A}$, or explicitly as

$$
\pi(x) \equiv \frac{1}{2 \sqrt{2}}\left(\begin{array}{cccc}
\pi^{3}(x) & \pi^{1}(x)-i \pi^{2}(x) & 0 & \pi^{5}(x)-i \pi^{4}(x) \\
\pi^{1}(x)+i \pi^{2}(x) & -\pi^{3}(x) & i \pi^{4}(x)-\pi^{5}(x) & 0 \\
0 & -i \pi^{4}(x)-\pi^{5}(x) & \pi^{3}(x) & \pi^{1}(x)+i \pi^{2}(x) \\
i \pi^{4}(x)+\pi^{5}(x) & 0 & \pi^{1}(x)-i \pi^{2}(x) & -\pi^{3}(x)
\end{array}\right) .
$$

The maximal $\mathrm{SO}(4) \sim \mathrm{SU}(2)_{L} \times \mathrm{SU}(2)_{R}$ subgroup of the unbroken $\mathrm{Sp}(4)$ can be chosen to be generated by

$$
\begin{aligned}
& T_{L}^{1}=\frac{1}{2}\left(\begin{array}{llll}
0 & 0 & 1 & 0 \\
0 & 0 & 0 & 0 \\
1 & 0 & 0 & 0 \\
0 & 0 & 0 & 0
\end{array}\right), \quad T_{L}^{2}=\frac{1}{2}\left(\begin{array}{cccc}
0 & 0 & -i & 0 \\
0 & 0 & 0 & 0 \\
i & 0 & 0 & 0 \\
0 & 0 & 0 & 0
\end{array}\right), \quad T_{L}^{3}=\frac{1}{2}\left(\begin{array}{cccc}
1 & 0 & 0 & 0 \\
0 & 0 & 0 & 0 \\
0 & 0 & -1 & 0 \\
0 & 0 & 0 & 0
\end{array}\right), \\
& T_{R}^{1}=\frac{1}{2}\left(\begin{array}{llll}
0 & 0 & 0 & 0 \\
0 & 0 & 0 & 1 \\
0 & 0 & 0 & 0 \\
0 & 1 & 0 & 0
\end{array}\right), \quad T_{R}^{2}=\frac{1}{2}\left(\begin{array}{cccc}
0 & 0 & 0 & 0 \\
0 & 0 & 0 & -i \\
0 & 0 & 0 & 0 \\
0 & i & 0 & 0
\end{array}\right), \quad T_{R}^{3}=\frac{1}{2}\left(\begin{array}{cccc}
0 & 0 & 0 & 0 \\
0 & 1 & 0 & 0 \\
0 & 0 & 0 & 0 \\
0 & 0 & 0 & -1
\end{array}\right) .
\end{aligned}
$$


The $T_{L}$ generators satisfy the $\mathrm{SU}(2)_{L}$ algebra $\left[T_{L}^{i}, T_{L}^{j}\right]=i \epsilon^{i j k} T_{L}^{k}$, and similarly $\left[T_{R}^{i}, T_{R}^{j}\right]=i \epsilon^{i j k} T_{R}^{k}$, while $\left[T_{L}^{A}, T_{R}^{B}\right]=0$. These generators being all unbroken (in a vacuum aligned with $\Omega$ ), this is the natural choice of embedding of the $\mathrm{SO}(4)$ symmetries of the Higgs field in the context of composite Higgs.

The same model can be used also to describe traditional technicolor. In this case, the embedding of the Standard Model symmetries is based on the natural choice of generators of $\mathrm{SO}(4)^{t} \sim \mathrm{SU}(2)_{L}^{t} \times \mathrm{SU}(2)_{R}^{t}$ as follows:

$$
\begin{aligned}
& t_{L}^{1}=\frac{1}{2}\left(\begin{array}{llll}
0 & 1 & 0 & 0 \\
1 & 0 & 0 & 0 \\
0 & 0 & 0 & 0 \\
0 & 0 & 0 & 0
\end{array}\right), \quad t_{L}^{2}=\frac{1}{2}\left(\begin{array}{cccc}
0 & -i & 0 & 0 \\
i & 0 & 0 & 0 \\
0 & 0 & 0 & 0 \\
0 & 0 & 0 & 0
\end{array}\right), \quad t_{L}^{3}=\frac{1}{2}\left(\begin{array}{cccc}
1 & 0 & 0 & 0 \\
0 & -1 & 0 & 0 \\
0 & 0 & 0 & 0 \\
0 & 0 & 0 & 0
\end{array}\right), \\
& t_{R}^{1}=-\frac{1}{2}\left(\begin{array}{llll}
0 & 0 & 0 & 0 \\
0 & 0 & 0 & 0 \\
0 & 0 & 0 & 1 \\
0 & 0 & 1 & 0
\end{array}\right), \quad t_{R}^{2}=-\frac{1}{2}\left(\begin{array}{cccc}
0 & 0 & 0 & 0 \\
0 & 0 & 0 & 0 \\
0 & 0 & 0 & -i \\
0 & 0 & i & 0
\end{array}\right), \quad t_{R}^{3}=-\frac{1}{2}\left(\begin{array}{cccc}
0 & 0 & 0 & 0 \\
0 & 0 & 0 & 0 \\
0 & 0 & 1 & 0 \\
0 & 0 & 0 & -1
\end{array}\right) .
\end{aligned}
$$

In this case, one finds that (with the vacuum aligned with $\Omega$ ) the breaking $\mathrm{SU}(2)_{L}^{t} \times$ $\mathrm{SU}(2)_{R}^{t} \rightarrow \mathrm{SU}(2)_{V}^{t}$ emerges, and the unbroken generators are $t_{V}^{A}=\left(t_{L}^{A}+t_{R}^{A T}\right)$, or explicitly:

$$
\begin{aligned}
& t_{V}^{1}=\frac{1}{2}\left(\begin{array}{cccc}
0 & 1 & 0 & 0 \\
1 & 0 & 0 & 0 \\
0 & 0 & 0 & -1 \\
0 & 0 & -1 & 0
\end{array}\right)=\sqrt{2} T^{13}, \quad t_{V}^{2}=\frac{1}{2}\left(\begin{array}{cccc}
0 & -i & 0 & 0 \\
i & 0 & 0 & 0 \\
0 & 0 & 0 & -i \\
0 & 0 & i & 0
\end{array}\right)=\sqrt{2} T^{8} \\
& t_{V}^{3}=\frac{1}{2}\left(\begin{array}{cccc}
1 & 0 & 0 & 0 \\
0 & -1 & 0 & 0 \\
0 & 0 & -1 & 0 \\
0 & 0 & 0 & 1
\end{array}\right)=\sqrt{2} T^{14} .
\end{aligned}
$$

The normalization is $\operatorname{Tr} t_{V}^{A} t_{V}^{B}=\delta^{A B}$, as in this case we are writing the generators in the bifundamental representation.

The unbroken $\mathrm{U}(1)_{B}^{t}$ associated with baryon number is generated by $T^{15}=\frac{1}{\sqrt{2}}\left(T_{L}^{3}+\right.$ $T_{R}^{3}$ ), while the anomalous axial $\mathrm{U}(1)_{A}$ is generated by

$$
T_{A}=\frac{1}{2 \sqrt{2}}\left(\begin{array}{llll}
1 & 0 & 0 & 0 \\
0 & 1 & 0 & 0 \\
0 & 0 & 1 & 0 \\
0 & 0 & 0 & 1
\end{array}\right)
$$




\section{Fit results of renormalized parameters}

In this appendix, we demonstrate how the fits in section 3.5 work by showing the renormalized parameters in eq. (3.7) along with the fit results of eq. (3.13) in the two-dimentional slices of the measured and lattice parameters. See figure 12 and figure 13 for the fermion and gauge anisotropies, and see figure 14 for the squared mass of pseudoscalar meson $M_{\mathrm{PS}}^{2}$.
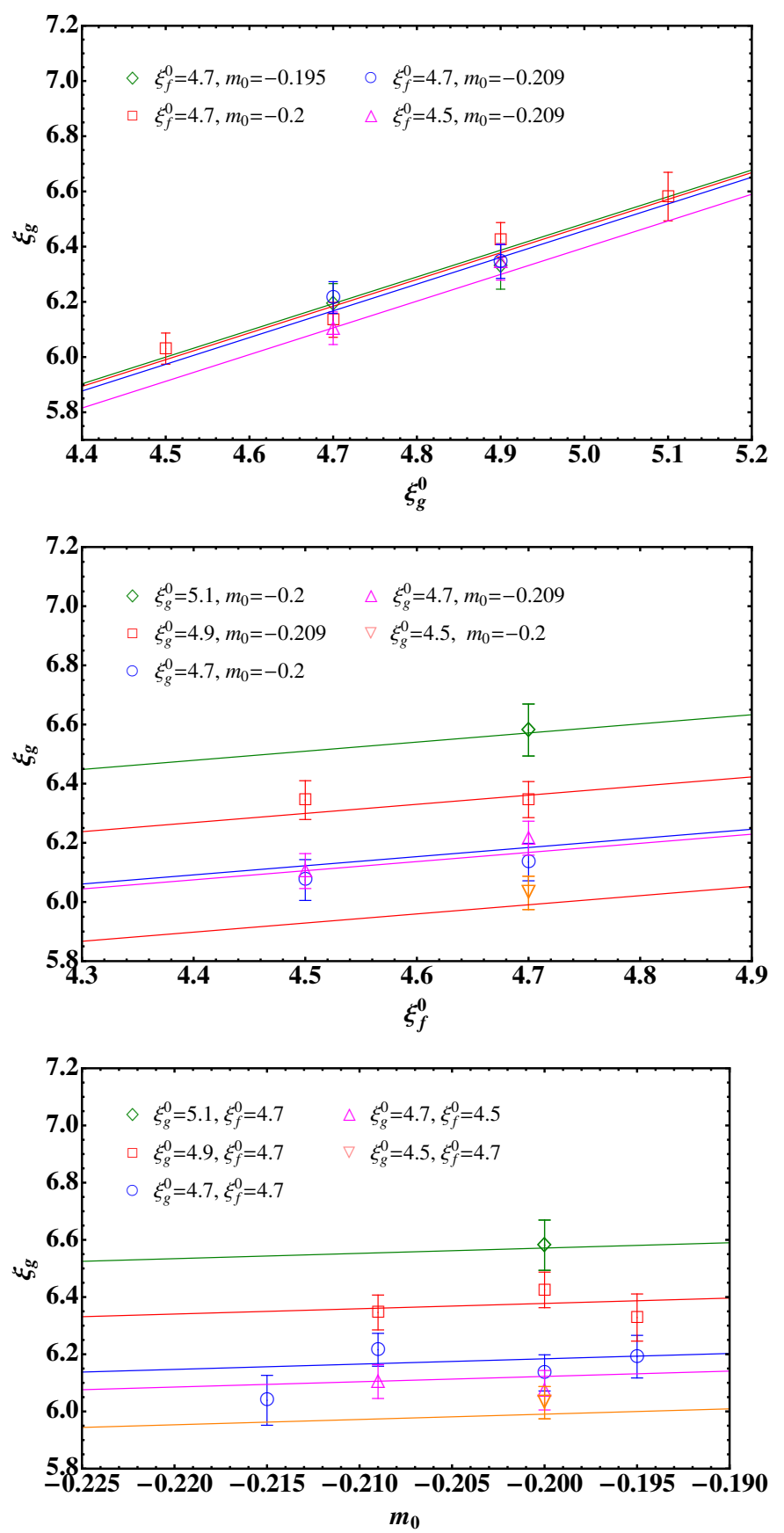

Figure 12. Gauge anisotropy $\xi_{g}$ as a function of $\xi_{g}^{0}, \xi_{f}^{0}$, and $m_{0}$. The solid lines denote the fit functions in eq. (3.7) where the coefficients are given by eq. (3.13). 

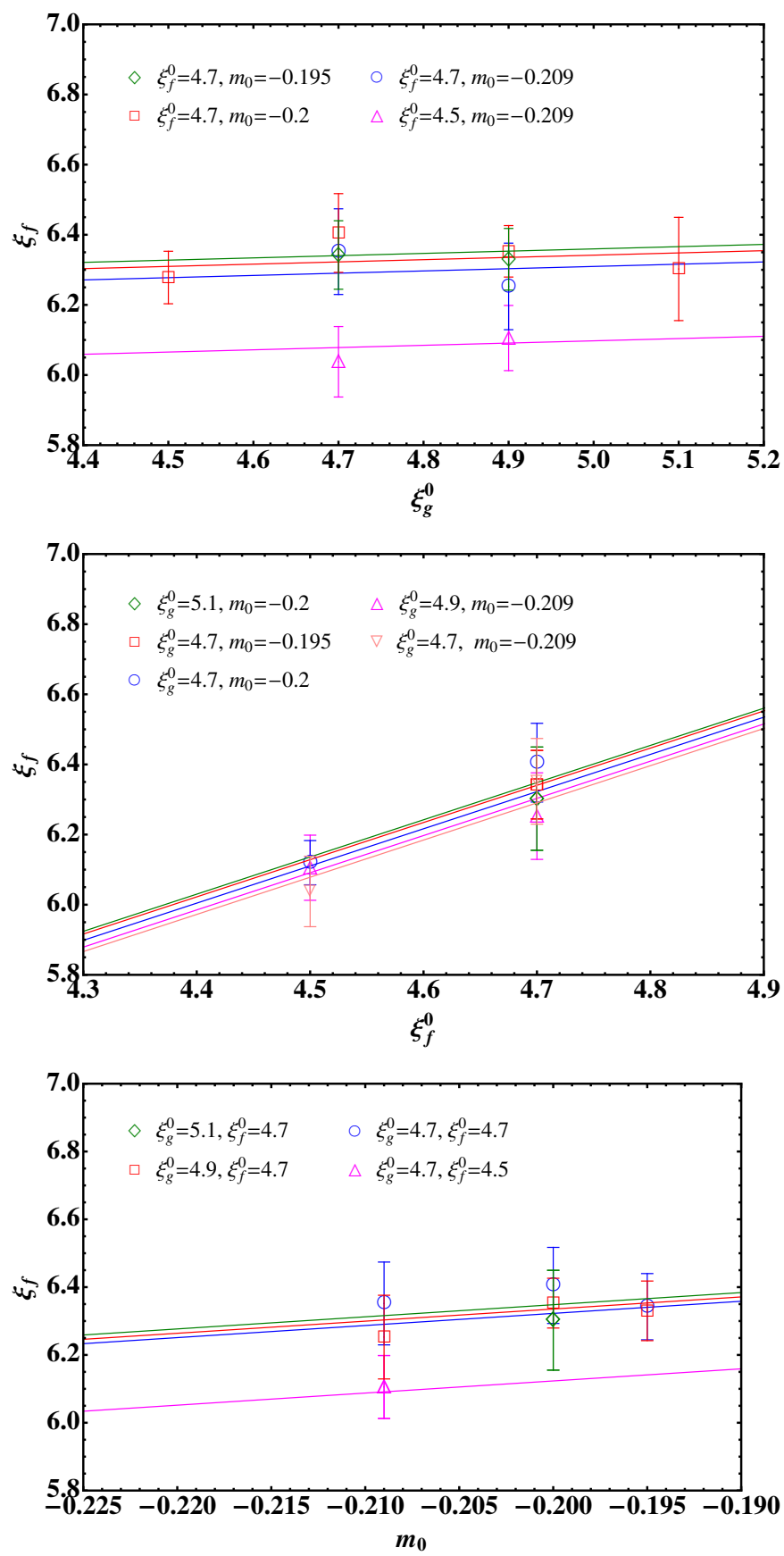

Figure 13. Fermion anisotropy $\xi_{f}$ as a function of $\xi_{g}^{0}$, $\xi_{f}^{0}$, and $m_{0}$. The solid lines denote the fit functions in eq. (3.7) where the coefficients are given by eq. (3.13). 

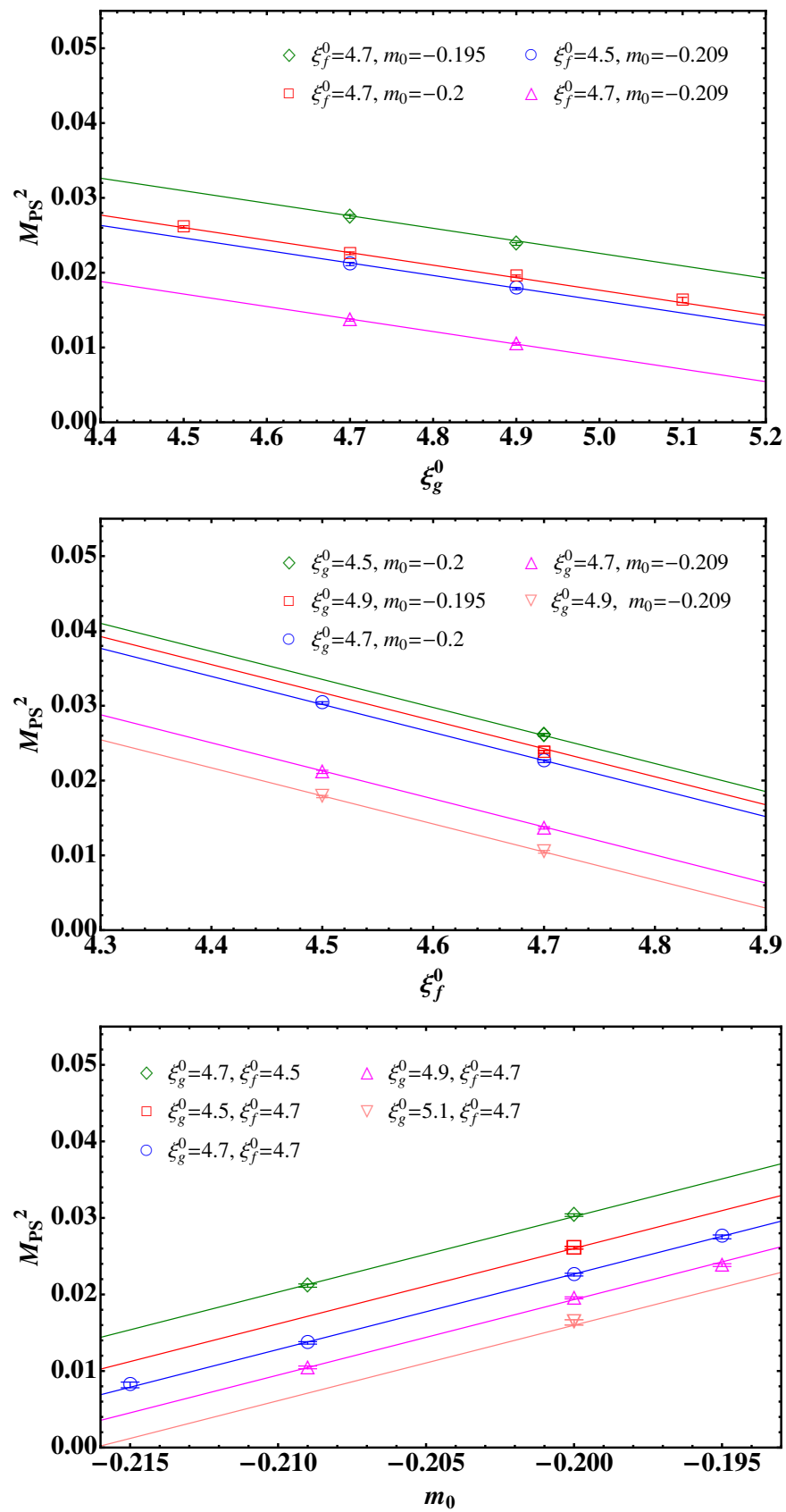

Figure 14. Squared mass of a pseudoscalar meson $M_{\mathrm{PS}}^{2}$ as a function of $\xi_{g}^{0}$, $\xi_{f}^{0}$, and $m_{0}$. The solid lines denote the fit functions in eq. (3.7) where the coefficients are given by eq. (3.13). 
Open Access. This article is distributed under the terms of the Creative Commons Attribution License (CC-BY 4.0), which permits any use, distribution and reproduction in any medium, provided the original author(s) and source are credited.

\section{References}

[1] K. Holland, M. Pepe and U.J. Wiese, The deconfinement phase transition of $\mathrm{Sp}(2)$ and $\mathrm{Sp}(3)$ Yang-Mills theories in $(2+1)$-dimensions and $(3+1)$-dimensions, Nucl. Phys. B 694 (2004) 35 [hep-lat/0312022] [INSPIRE].

[2] S. Hands, S. Kim and J.-I. Skullerud, Deconfinement in dense 2-color QCD, Eur. Phys. J. C 48 (2006) 193 [hep-lat/0604004] [INSPIRE].

[3] S. Hands, S. Kim and J.-I. Skullerud, Quark matter in QC(2)D, Eur. Phys. J. A 31 (2007) 787 [nucl-th/0609012] [INSPIRE].

[4] S. Weinberg, Implications of dynamical symmetry breaking: an addendum, Phys. Rev. D 19 (1979) 1277 [INSPIRE].

[5] L. Susskind, Dynamics of spontaneous symmetry breaking in the Weinberg-Salam theory, Phys. Rev. D 20 (1979) 2619 [inSPIRE].

[6] S. Weinberg, Implications of dynamical symmetry breaking, Phys. Rev. D 13 (1976) 974 [INSPIRE].

[7] R.S. Chivukula, Lectures on technicolor and compositeness, hep-ph/0011264 [INSPIRE].

[8] K. Lane, Two lectures on technicolor, hep-ph/0202255 [inSPIRE].

[9] C.T. Hill and E.H. Simmons, Strong dynamics and electroweak symmetry breaking, Phys. Rept. 381 (2003) 235 [Erratum ibid. 390 (2004) 553] [hep-ph/0203079] [INSPIRE].

[10] A. Martin, Predicted signals at the LHC from technicolor: Erice lecture, Subnucl. Ser. 46 (2011) 135 [arXiv: 0812.1841] [INSPIRE].

[11] F. Sannino, Conformal dynamics for TeV physics and cosmology, Acta Phys. Polon. B 40 (2009) 3533 [arXiv: 0911.0931] [InSPIRE].

[12] M. Piai, Lectures on walking technicolor, holography and gauge/gravity dualities, Adv. High Energy Phys. 2010 (2010) 464302 [arXiv: 1004.0176] [INSPIRE].

[13] M.E. Peskin and T. Takeuchi, Estimation of oblique electroweak corrections, Phys. Rev. D 46 (1992) 381 [INSPIRE].

[14] R. Lewis, C. Pica and F. Sannino, Light asymmetric dark matter on the lattice: SU(2) technicolor with two fundamental flavors, Phys. Rev. D 85 (2012) 014504 [arXiv:1109.3513] [INSPIRE].

[15] G. Cacciapaglia and F. Sannino, Fundamental composite (Goldstone) Higgs dynamics, JHEP 04 (2014) 111 [arXiv: 1402.0233] [INSPIRE].

[16] A. Hietanen, R. Lewis, C. Pica and F. Sannino, Fundamental composite Higgs dynamics on the lattice: SU(2) with two flavors, JHEP 07 (2014) 116 [arXiv:1404.2794] [INSPIRE].

[17] A. Arbey, G. Cacciapaglia, H. Cai, A. Deandrea, S. Le Corre and F. Sannino, Fundamental composite electroweak dynamics: status at the LHC, Phys. Rev. D 95 (2017) 015028 [arXiv: 1502.04718] [INSPIRE]. 
[18] R. Arthur, V. Drach, A. Hietanen, C. Pica and F. Sannino, SU(2) gauge theory with two fundamental flavours: scalar and pseudoscalar spectrum, arXiv:1607.06654 [INSPIRE].

[19] E. Katz, A.E. Nelson and D.G.E. Walker, The intermediate Higgs, JHEP 08 (2005) 074 [hep-ph/0504252] [INSPIRE].

[20] P. Lodone, Vector-like quarks in a 'composite' Higgs model, JHEP 12 (2008) 029 [arXiv: 0806.1472] [INSPIRE].

[21] B. Gripaios, A. Pomarol, F. Riva and J. Serra, Beyond the minimal composite Higgs model, JHEP 04 (2009) 070 [arXiv: 0902.1483] [INSPIRE].

[22] J. Barnard, T. Gherghetta and T.S. Ray, UV descriptions of composite Higgs models without elementary scalars, JHEP 02 (2014) 002 [arXiv:1311.6562] [INSPIRE].

[23] G. Ferretti and D. Karateev, Fermionic UV completions of composite Higgs models, JHEP 03 (2014) 077 [arXiv: 1312.5330] [INSPIRE].

[24] L. Vecchi, A dangerous irrelevant UV-completion of the composite Higgs, JHEP 02 (2017) 094 [arXiv: 1506.00623] [INSPIRE].

[25] G. Ferretti, Gauge theories of partial compositeness: scenarios for Run-II of the LHC, JHEP 06 (2016) 107 [arXiv: 1604.06467] [INSPIRE].

[26] A. Agugliaro, O. Antipin, D. Becciolini, S. De Curtis and M. Redi, UV complete composite Higgs models, Phys. Rev. D 95 (2017) 035019 [arXiv:1609.07122] [InSPIRE].

[27] G. Panico and A. Wulzer, The composite Nambu-Goldstone Higgs, Lect. Notes Phys. 913 (2016) 1 [arXiv: 1506.01961].

[28] D.B. Kaplan, H. Georgi and S. Dimopoulos, Composite Higgs scalars, Phys. Lett. B 136 (1984) 187 [INSPIRE].

[29] M.E. Peskin, The alignment of the vacuum in theories of technicolor, Nucl. Phys. B 175 (1980) 197 [INSPIRE].

[30] QCD-TARO collaboration, P. de Forcrand et al., Meson correlators in finite temperature lattice QCD, Phys. Rev. D 63 (2001) 054501 [hep-lat/0008005] [INSPIRE].

[31] M. Cheng et al., Meson screening masses from lattice QCD with two light and the strange quark, Eur. Phys. J. C 71 (2011) 1564 [arXiv:1010.1216] [INSPIRE].

[32] G. Aarts, C. Allton, S. Hands, B. Jäger, C. Praki and J.-I. Skullerud, Nucleons and parity doubling across the deconfinement transition, Phys. Rev. D 92 (2015) 014503 [arXiv: 1502.03603] [INSPIRE].

[33] T. Alanne, H. Gertov, A. Meroni and F. Sannino, Vacuum alignment with and without elementary scalars, Phys. Rev. D 94 (2016) 075015 [arXiv: 1608.07442] [INSPIRE].

[34] J. Schechter and Y. Ueda, General treatment of the breaking of chiral symmetry and scale invariance in the SU(3) $\sigma$-model, Phys. Rev. D 3 (1971) 2874 [Erratum ibid. D 8 (1973) 987] [INSPIRE].

[35] L.-H. Chan and R.W. Haymaker, Closed-loop corrections to the $\mathrm{SU}(3) \times \mathrm{SU}(3) \sigma$-model one- and two-point functions, Phys. Rev. D 7 (1973) 402 [INSPIRE].

[36] L.-H. Chan and R.W. Haymaker, Renormalization of the $\mathrm{SU}(3) \times \mathrm{SU}(3) \sigma$-model, Phys. Rev. D 7 (1973) 415 [INSPIRE].

[37] G. 't Hooft, How instantons solve the U(1) problem, Phys. Rept. 142 (1986) 357 [INSPIRE]. 
[38] M. Napsuciale, Scalar meson masses and mixing angle in a $\mathrm{U}(3) \times \mathrm{U}(3)$ linear $\sigma$-model, hep-ph/9803396 [INSPIRE].

[39] B.B. Brandt, A. Francis, H.B. Meyer, O. Philipsen, D. Robaina and H. Wittig, On the strength of the $U_{A}(1)$ anomaly at the chiral phase transition in $N_{f}=2$ QCD, JHEP 12 (2016) 158 [arXiv : 1608.06882] [INSPIRE].

[40] S. Chandrasekharan and A.C. Mehta, Effects of the anomaly on the two-flavor QCD chiral phase transition, Phys. Rev. Lett. 99 (2007) 142004 [arXiv:0705.0617] [INSPIRE].

[41] M. Bando, T. Kugo, S. Uehara, K. Yamawaki and T. Yanagida, Is $\rho$ meson a dynamical gauge boson of hidden local symmetry?, Phys. Rev. Lett. 54 (1985) 1215 [INSPIRE].

[42] R. Casalbuoni, S. De Curtis, D. Dominici and R. Gatto, Effective weak interaction theory with possible new vector resonance from a strong Higgs sector, Phys. Lett. B 155 (1985) 95 [INSPIRE].

[43] M. Bando, T. Kugo and K. Yamawaki, Nonlinear realization and hidden local symmetries, Phys. Rept. 164 (1988) 217 [INSPIRE].

[44] M. Harada and K. Yamawaki, Hidden local symmetry at loop: A New perspective of composite gauge boson and chiral phase transition, Phys. Rept. 381 (2003) 1 [hep-ph/0302103] [INSPIRE].

[45] H. Georgi, Vector realization of chiral symmetry, Nucl. Phys. B 331 (1990) 311 [INSPIRE].

[46] M. Piai, A. Pierce and J.G. Wacker, Composite vector mesons from QCD to the little Higgs, hep-ph/0405242 [INSPIRE].

[47] E. Bennett, J.W. Lee, C.-J. D. Lin, B. Lucini, M. Piai and D. Vadacchino, in preparation.

[48] R.G. Edwards, B. Joo and H.-W. Lin, Tuning for three-flavors of anisotropic clover fermions with stout-link smearing, Phys. Rev. D 78 (2008) 054501 [arXiv:0803.3960] [INSPIRE].

[49] L. Del Debbio, A. Patella and C. Pica, Higher representations on the lattice: Numerical simulations. SU(2) with adjoint fermions, Phys. Rev. D 81 (2010) 094503 [arXiv: 0805.2058] [INSPIRE].

[50] R. Morrin, A.O. Cais, M. Peardon, S.M. Ryan and J.-I. Skullerud, Dynamical QCD simulations on anisotropic lattices, Phys. Rev. D 74 (2006) 014505 [hep-lat/0604021] [INSPIRE].

[51] T.R. Klassen, The anisotropic Wilson gauge action, Nucl. Phys. B 533 (1998) 557 [hep-lat/9803010] [INSPIRE].

[52] CP-PACS collaboration, T. Umeda et al., Two flavors of dynamical quarks on anisotropic lattices, Phys. Rev. D 68 (2003) 034503 [hep-lat/0302024] [INSPIRE].

[53] B. Bolder et al., A high precision study of the $Q \bar{Q}$ potential from Wilson loops in the regime of string breaking, Phys. Rev. D 63 (2001) 074504 [hep-lat/0005018] [INSPIRE].

[54] S. Borsányi et al., QCD thermodynamics with dynamical overlap fermions, Phys. Lett. B 713 (2012) 342 [arXiv:1204.4089] [INSPIRE].

[55] G. Aarts, C. Allton, A. Amato, P. Giudice, S. Hands and J.-I. Skullerud, Electrical conductivity and charge diffusion in thermal QCD from the lattice, JHEP 02 (2015) 186 [arXiv: 1412.6411] [INSPIRE]. 
[56] O. Kaczmarek, F. Karsch, P. Petreczky and F. Zantow, Heavy quark anti-quark free energy and the renormalized Polyakov loop, Phys. Lett. B 543 (2002) 41 [hep-lat/0207002] [INSPIRE].

[57] S. Gupta, K. Huebner and O. Kaczmarek, Renormalized Polyakov loops in many representations, Phys. Rev. D 77 (2008) 034503 [arXiv:0711.2251] [INSPIRE].

[58] P.A. Boyle, A. Juttner, C. Kelly and R.D. Kenway, Use of stochastic sources for the lattice determination of light quark physics, JHEP 08 (2008) 086 [arXiv:0804.1501] [INSPIRE].

[59] D. Weingarten, Mass inequalities for QCD, Phys. Rev. Lett. 51 (1983) 1830 [InSPIRE].

[60] C.E. Detar and J.B. Kogut, Measuring the hadronic spectrum of the quark plasma, Phys. Rev. D 36 (1987) 2828 [INSPIRE]. 